\title{
The CoPhyLab comet-simulation chamber
}

Cite as: Rev. Sci. Instrum. 92, 115102 (2021); https://doi.org/10.1063/5.0057030

Submitted: 17 May 2021 • Accepted: 15 September 2021 • Published Online: 02 November 2021

(D) C. Kreuzig, (D) G. Kargl, (D) A. Pommerol, et al.

\section{COLLECTIONS}

This paper was selected as Featured
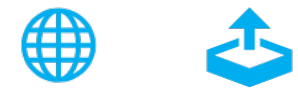

\section{ARTICLES YOU MAY BE INTERESTED IN}

Estimation of the information contained in the visible matter of the universe

AIP Advances 11, 105317 (2021); https://doi.org/10.1063/5.0064475

Total-reflection high-energy positron diffractometer at NEPOMUC - Instrumentation, simulation, and first measurements

Review of Scientific Instruments 92, 115103 (2021); https://doi.org/10.1063/5.0062412

Integrated atomic force microscopy and x-ray irradiation for in situ characterization of radiation-induced processes

Review of Scientific Instruments 92, 113701 (2021); https://doi.org/10.1063/5.0054646

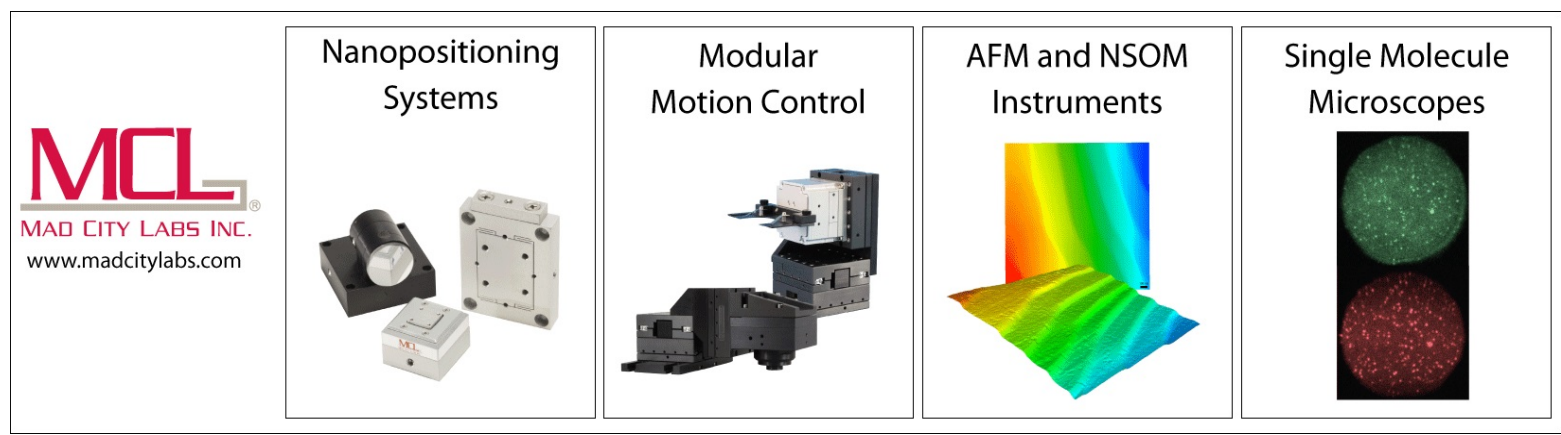




\title{
The CoPhyLab comet-simulation chamber
}

Cite as: Rev. Sci. Instrum. 92, 115102 (2021); doi: 10.1063/5.0057030

Submitted: 17 May 2021 - Accepted: 15 September 2021 •

Published Online: 2 November 2021

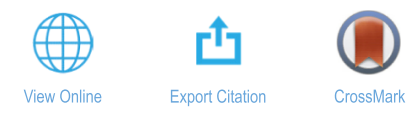

\author{
C. Kreuzig,, ${ }^{1, a)}$ (D) G. Kargl, ${ }^{2}$ (D) A. Pommerol, ${ }^{3}$ (D) J. Knollenberg, ${ }^{4}$ A. Lethuillier, ${ }^{7}$ N. S. Molinski, ${ }^{1}$ T. Gilke, \\ D. Bischoff, ${ }^{1}$ (D) C. Feller, ${ }^{3}$ E. Kührt, ${ }^{4}$ (D) H. Sierks, ${ }^{5}$ N. Hänni, ${ }^{3}$ (D) H. Capelo, ${ }^{3}$ C. Güttler, ${ }^{5}$ D. Haack, ${ }^{4}$ K. Otto, \\ E. Kaufmann, ${ }^{2}$ M. Schweighart, ${ }^{2}$ W. Macher, ${ }^{2}$ P. Tiefenbacher, ${ }^{2}$ B. Gundlach, ${ }^{1}$ and J. Blum ${ }^{7}$ (iD)
}

\begin{abstract}
AFFILIATIONS
${ }^{1}$ Institut für Geophysik und extraterrestrische Physik (IGeP), TU Braunschweig, Mendelssohnstr. 3, 38106 Braunschweig, Germany

${ }^{2}$ Space Research Institute, Austrian Academy of Science, Schmiedlstraße 6, 8042 Graz, Austria

${ }^{3}$ Physics Institute for Space Research and Planetary Science, University of Bern, Sidlerstrasse 5, 3012 Bern, Switzerland

${ }^{4}$ Deutsches Zentrum für Luft- und Raumfahrt, Rutherfordstraße 2, 12489 Berlin-Adlershof, Germany

${ }^{5}$ Max-Planck-Institut für Sonnensystemforschung, Justus-von-Liebig-Weg 3, 37077 Göttingen, Germany
\end{abstract}

a) Author to whom correspondence should be addressed: c.kreuzig@tu-braunschweig.de

\begin{abstract}
The Comet Physics Laboratory (CoPhyLab) is an international research program to study the physical properties of cometary analog materials under simulated space conditions. The project is dedicated to studying, with the help of multiple instruments and the different expertise and background from the different partners, the physics of comets, including the processes inside cometary nuclei, the activity leading to the ejection of dust and gas, and the sub-surface and surface evolution of cometary nuclei when exposed to solar illumination. CoPhyLab will provide essential information on the formation and evolution of comets and insights into the origins of primitive Solar System bodies. To this end, we constructed a new laboratory that hosts several small-scale experiments and a large-scale comet-simulation chamber (L-Chamber). This chamber has been designed and constructed to host ice-dust samples with a diameter of up to $250 \mathrm{~mm}$ and a variable height between 100 and $300 \mathrm{~mm}$. The cometary-analog samples will be kept at temperatures below $120 \mathrm{~K}$ and pressures around $10^{-6}$ mbar to ensure cometarylike conditions. In total, 14 different scientific instruments are attached to the L-Chamber to study the temporal evolution of the physical properties of the sample under different insolation conditions. Due to the implementation of a scale inside the L-Chamber that can measure weight changes of the samples with high precision, the cooling system is mechanically decoupled from the sample holder and cooling of the samples occurs by radiation only. The constructed chamber allows us to conduct uninterrupted experiments at low temperatures and pressures up to several weeks.
\end{abstract}

Published under an exclusive license by AIP Publishing. https://doi.org/10.1063/5.0057030

\section{INTRODUCTION}

Comets are small dark bodies often located far away from the Sun in the Oort cloud or in the Kuiper belt and therefore are difficult to observe. However, some of them were deflected by gravitational influences and are presently on highly elliptic orbits around the Sun. On their orbits, comets come close to the Sun and are responsible for the occurrence of the comet tails, which can be easily visible from the Earth. The dust tail is caused by the sublimation of ices, heated by the Sun's radiation, which then drags dust aggregates away from the surface. Since cometary tails are clearly visible from the Earth, there has always been interest in understanding this phenomenon.
Today, the aim of comet research is not only to understand the formation of the cometary comae but also to investigate the origins of our Solar System, since comets presumably are among the most pristine objects, as they were stored far away from the Sun most of their lifetime and have experienced none or only few collisions. Currently, two different formation scenarios are debated in the cometary science community, ${ }^{1}$ namely, the gravitational collapse scenario $^{2-5}$ and the mass transfer scenario, ${ }^{6-8}$ which lead to different internal structures of the resulting body. The aim of Comet Physics Laboratory (CoPhyLab) is to investigate samples corresponding to the proposed planetesimal formation scenarios and thereby to determine which scenario has led to the formation of comets. 
Our understanding of comets has been significantly improved by several space missions in the last few decades, most recently in 2014-2016, by the Rosetta mission to comet 67P/Churyumov-Gerasimenko. These missions were supported by multiple theoretical models and also by large observational campaigns. However, the support from ground-based laboratory experiments has been limited, although they can provide deeper understanding about the physics of comets.

The first laboratory comet-simulation experiments were performed by Soviet scientists in the 1970s. They investigated the sublimation of water ice mixed with minerals and organic materials. In these experiments, a porous crust of non-volatile materials formed as the ice sublimated and some fragments were ejected. ${ }^{9}$ Later in the 1980s, similar experiments were performed at the Jet Propulsion Laboratory (JPL), showing that some minerals and organic materials can form filamentary sublimate residues with interesting physical and textural properties. ${ }^{10,11}$ After the successful Giotto mission in 1986, the KOmeten-SImulation (KOSI) experiments were performed at the Deutsches Zentrum für Luft- und Raumfahrt (DLR). This is the most comprehensive study so far and it provided interesting insights into morphological and physical properties of illuminated ice-dust samples under high-vacuum conditions. ${ }^{12,13}$ At JPL, another campaign was carried out, which was also based on studying irradiated ice-dust mixtures. The main focus of this experiment was placed on the penetration of the heat wave and the release of dust. The results from this campaign are unfortunately not generally available in the literature. Both experiment campaigns suffered from the fact that too many parameters, such as the sample ingredients, sample preparation, and experiment conditions, were all changed at the same time. This increased the difficulty to interpret the data because a change in behavior cannot be related to a single change in the experiment. Furthermore, the knowledge gained during the
Rosetta mission shows that the samples used in these campaigns did not represent the cometary composition in terms of the dust-to-ice ratio. Most experiments used very icy samples with a low dust-to-ice ratio; ${ }^{14}$ however, new data suggest that comets may consist primarily of dust ${ }^{15}$ with dust-to-ice mass ratios between 3:1 and 9:1. . $^{16,17}$

In the framework of the CoPhyLab project, we aim to avoid these problems by concentrating on the understanding of the physical processes that lead to the ejection of dust from comets and not trying to replicate a comet with all its components, internal structures, and surface morphologies. The CoPhyLab project is organized in three stages: the $S, M$, and $L$ phases. In the $S$ phase, we perform small-scale experiments with only a single component to investigate isolated physical properties. The aim of this phase is also to characterize the properties of the ingredients used in later phases for our CoPhyLab dust samples. This non-volatile comet analog material is our standard dust mixture that we will use in later experiments (Lethuillier et al. in prep). During the $\mathrm{M}$ phase of this project, the complexity of the experiments is increased by adding more ingredients into the mixtures and by performing experiments that combine different measurement techniques. In addition, this phase is dedicated to bring the instruments from the different project partners together for their use in the L-Chamber. In the L phase, we will then perform long-term experiments over multiple weeks with the whole suite of instruments installed on the L-Chamber.

The L-Chamber, its surrounding infrastructure, and the installed instruments are presented in this paper. In Sec. II, we describe the scientific objectives of the CoPhyLab project, which we structure in five groups, namely, mechanical properties, temperatures, morphology, gas and dust emission, and optical properties, respectively. Table I summarizes all instruments we selected for each scientific objective. After that, Sec. III discusses the design and construction of the CoPhyLab comet-simulation

TABLE I. Summary of scientific objectives that shall be investigated in the CoPhyLab L-Chamber. For each objective, a list of appropriate instruments that will be utilized is provided. For each instrument, a link to the describing section in this paper is given.

\begin{tabular}{|c|c|c|}
\hline Scientific objective & Instruments & Description \\
\hline \multirow[t]{5}{*}{ Mechanical properties } & Penetrometer (standard) & Sec. IV A 1 \\
\hline & Penetrometer (BDT) & Sec. IV A 2 \\
\hline & Infrared camera & Sec. IV B 1 \\
\hline & MARA & Sec. IV B 2 \\
\hline & Scale & Sec. IV D 2 \\
\hline \multirow[t]{4}{*}{ Temperatures } & Mass spectrometer & Sec. IV D 3 \\
\hline & Pt 100 temperature sensors & Sec. IV B 3 \\
\hline & Heatstrip & Sec. IV B 4 \\
\hline & Thermoneedles & Sec. IV B 5 \\
\hline \multirow{2}{*}{ Surface morphology } & Morphology change trigger and observation system & Sec. IV C 1 \\
\hline & Mobile stereo imaging system & Sec. IV C 2 \\
\hline \multirow{3}{*}{ Gas and dust emission } & High-speed tracking cameras & Sec. IV D 1 \\
\hline & Scale & Sec. IV D 2 \\
\hline & Mass spectrometer & Sec. IV D 3 \\
\hline \multirow{3}{*}{ Optical properties } & Mobile hyperspectral imaging setup & Sec. IV E 1 \\
\hline & Mobile and modular visible imaging setup & Sec. IV E 2 \\
\hline & Optical fibers & Sec. IV E 3 \\
\hline
\end{tabular}


chamber (L-Chamber). In Sec. IV, the individual instruments, their location in the L-Chamber, and their supporting systems are presented. The sample preparation and transport to the chamber are described in Sec. V. The procedures required to operate the chamber are explained in Sec. VI. Furthermore, the performance of the $\mathrm{L}$-Chamber is reviewed at the end of Sec. VI.

\section{SCIENTIFIC OBJECTIVES}

In Subsections II A-II E, we present an overview of the scientific objectives of the CoPhyLab project as well as the suite of instruments that will be used to achieve these goals. Table I summarizes this in a compact way.

\section{A. Mechanical properties}

In order to understand cometary activity, it is essential to determine the mechanical properties of cometary (analog) materials. ${ }^{18}$ These properties determine, e.g., the amount of force required to detach particles, aggregates, or agglomerates from the surface and also how much stress a structure can resist before it collapses. Furthermore, the mechanical properties of ice-dust mixtures can change by thermal metamorphism of the icy samples, ${ }^{19}$ and it is of major importance to measure these effects. In particular, we focus on measuring the compressive and tensile strengths of the material. The compressive strength will be measured by a penetrometer during long-term experiments with multiple day-and-night cycles. This will provide information about the temporal evolution of the overall compressive strength and on the possible formation of layers. The tensile strength will be derived indirectly by observing the collapse of artificial cliffs and other surface structures or through numerical simulations of observed dust activity. Furthermore, the tensile strength will be measured directly by utilizing the so-called Brazilian disk test. This method has been used by Gundlach et al. ${ }^{20}$ with pure water ice and silica dust and has been validated by comparing the results to other experiments. We already used this method in the framework of the CoPhyLab and other projects to measure the tensile strength of ice-dust mixtures, ${ }^{21}$ of several organic materials, ${ }^{22}$ and of highly compressed dust samples. ${ }^{23}$

\section{B. Temperatures}

The surface temperature is a very important parameter for the understanding of cometary activity. It determines the sublimation of sub-surface ices and thereby the dust ejection process. Hence, the temperature is one of the most important parameters required for the thermal modeling of comets and can change rapidly if the illumination source is switched off. The thermal properties of the surface of cometary nuclei have been rather well determined recently due to measurements from the Rosetta orbiter. The thermal properties of some of the individual components of primitive Solar System matter, such as water ice, $\mathrm{CO}_{2}$ ice, and carbonaceous chondrites, are known, ${ }^{24}$ and some work has been done to investigate the corresponding properties of porous dust samples. ${ }^{25}$ Granular samples have a relatively low thermal inertia because of their low heat conductivity caused by the high porosity. This means that most of the incoming energy is stored in the upper surface layers and thermal transport into the interior is inefficient and slow. If the temperature distribution inside the sample is modeled by applying the heat conduction equation together with its boundary conditions (see, e.g., the work of $\mathrm{Hu}$ et al. ${ }^{26}$ ), the measured temperature data can be used to constrain the thermal conductivity, a key parameter to describe the thermal behavior of the matter. Together with the density and heat capacity of the sample, other parameters such as the thermal diffusivity and the thermal inertia that are frequently used in the literature can be derived.

With CoPhyLab, we plan to use multiple instruments to measure the surface temperature as well as the temperature distribution inside the analog sample. An infrared camera and the MAscot RAdiometer (MARA) flight-spare sensor head will be used to determine the surface temperatures. While the infrared camera allows us to spatially resolve the surface temperature, the MARA sensor can operate at lower temperatures. In addition to these radiative temperature devices, an array of temperatures sensors will continuously monitor the internal temperature distribution of the samples. With the knowledge of the three-dimensional temperature distribution, we will be able to calibrate numerical heat-transfer models. On top of that, measuring the change in the sample's weight with the scale, the position and temperature of the sublimating ices can be determined.

\section{Surface morphology}

The surface morphology of comets on various spatial scales has been revealed by recent space missions as being highly diverse and variable in time and location. ${ }^{27-30}$ The main process determining the morphology of comets is the activity caused by outgassing of volatiles and dust emission when approaching the Sun. ${ }^{31}$ Among others, macroscopic morphological features related to cometary activity include fractures and polygonal networks, ${ }^{32}$ pits, ${ }^{33}$ debris deposits with boulders formed by collapsing cliffs, ${ }^{34}$ and smooth areas of redistributed particles. These features, for example, collapsed cliffs, can also be used to derive macroscopic physical properties, such as the tensile strength. ${ }^{35}$ On smaller scales, cometary outgassing may introduce surface roughness, ${ }^{36,37}$ which, in turn, influences remote-sensing observations including reflectance spectroscopy, photometric observations, and infrared radiometry.

Morphology on small and large scales will be investigated in the CoPhyLab experiments. Large morphological features, including artificial cliffs and large structures resembling boulders on comets, will be monitored by high-resolution video cameras inside and outside the simulation chamber to determine their morphological evolution. Small-scale changes in surface texture and morphology will be characterized by using high-resolution images and compared to observations made by space missions. Our aim is to relate morphological features and appearances of cometary analog materials in the laboratory to the evolution of cometary surfaces and constrain the physical parameters, such as the tensile strength or volatile content, by independent laboratory measurements. The scaling from the laboratory to the cometary environment is a challenging aspect for CoPhyLab. Due to the effect of gravity, pebbles of a $\mathrm{mm}$ in size would be in the cohesive regime on a comet, but on the Earth, the same pebbles will be dominated by gravitation. To realize a cohesive material in the laboratory, the grain size has to be scaled-down accordingly. In addition, the condition that activity can be observed changes under the effect of Earth's gravity. The gas pressure has to overcome the 
tensile strength of the material plus the weight of the overlying material, which is on the Earth much higher than on a comet. Hence, a higher gas pressure is required to lift off the particles/pebbles from the surface in the laboratory. ${ }^{21}$

\section{Gas and dust emission}

The emission of gas and dust is of major interest for the understanding of comets and their activity. Therefore, the outgassing rate of various volatile species will be measured by a system consisting of a mass spectrometer with a chopper and the in-vacuum scale that measures the sample mass over time with high precision. Using this system, we are able to measure the mass loss due to the ambient outgassing of the sample. The ejection of the solid material will be monitored by high-resolution cameras, which will allow the determination of the ejected solid mass. Additionally, three-dimensional Particle tracking velocimetry $(3 \mathrm{D}-\mathrm{PTV})^{38-42}$ will be used. The primary goal of 3D-PVT is to monitor the trajectories of the dust and ice ejecta. Such data yield constraints for important properties, such as density and velocity field of the underlying gas flow. Understanding the transport of dust from the inner to the outer coma is critical when matching high-resolution computer simulations with spacecraft measurements. ${ }^{43,44}$ The $3 \mathrm{D}-\mathrm{PTV}$ technique is adapted from flow measurement technology, which involves high-speed stereoscopic imaging of tracer particles that are coupled to a fluid flow. The derived velocity and acceleration vectors allow us to quantify the behavior of the underlying gas flow.

Dynamic measurements of the ejecta will be of importance when cross-referenced to the outgassing rate measured by the mass spectrometer (described in more detail in Sec. IV D 3), the volatile loss inferred from the spectra (see Sec. IV E), the mass loss measured by the scale (described in Sec. IV D 2), and the temperature flux measurements inside the sample (shown in Secs. IV B 3-IV B 5). Together, all these measurements will provide us with a full description of the advection-diffusion of heat and momentum both in and above the sample. Furthermore, readings from the scale will include ejecta that fall back to the sample surface, and the trajectory analysis will help address this potential source of bias in the mass-loss determination. Three-dimensional measurements will be particularly useful, since we can also check, for example, the isotropy of the outgassing flow, which is an assumption of the mass spectroscopy analysis but could be more complicated for evolved samples that may develop cracks and local inhomogeneities (see Sec. IV C).

\section{E. Optical properties}

Whether one is looking at a colloidal suspension, aerosols in the atmosphere of another planet, dust particles around a comet, or the particle surface of an atmosphereless celestial body, the way a medium scatters light is closely related to the basic physical and chemical properties of that medium. ${ }^{45-48}$ In particular, the reflectance varies with the ratio of wavelength to particle size and the viewing geometry. Such variations are determined by the composition and the structure of the medium. ${ }^{4-52}$

In the L-Chamber, we will perform spectroscopic and spectrophotometric measurements as well as tests of the inversion of reflectance mixing models. These measurements will be performed during the sublimation or in between sublimation sequences inside the L-Chamber under artificial solar irradiation. Those measurements have been tested previously by the Planetary Imaging Group (PIG) of the Bern University, which specialized in the remotesensing of icy and active planetary surfaces. ${ }^{5}$

\section{OVERVIEW OF THE CHAMBER}

The motivation for the construction of the L-Chamber was to set up an experiment that allows multiple measurements at the same time with the same sample. All instruments should be independent from each other so that experiments can be performed with any selection of instruments. This strategy provides the option of designing experiment campaigns without having to compromise on instrument selection.

\section{A. Chamber construction}

The construction of the L-Chamber had to fulfill many requirements. The first requirement was the space needed for all the different instruments mounted on or inside the vacuum chamber. This also includes the sample cooling system (see Sec. III D), the cooling shield (see Sec. III C), the scale (see Sec. IV D 2), and the sample itself. The second requirement was that all instruments have to be operated simultaneously. Hence, individual flanges for all instruments and for the operation of the L-Chamber were designed appropriately. This further allows any combination of instruments to be selected for a certain experimental run or time slot. The third requirement was that the chamber had to be transported by a standard lift truck through a standard sized door. Hence, the chamber dimension were optimized with respect to these requirements. The weight of the chamber was not a major constraint because a crane was used to lift the individual parts of the chamber into their final position. In order to keep the cost of the chamber as low as possible, the chamber is composed of standard parts whenever possible.

The L-Chamber is manufactured out of stainless steel, possesses a rectangular shape and an inner volume of $1530 \times 700 \times 700 \mathrm{~mm}^{3}$. The main advantage of this shape is that all flanges can be welded onto flat surfaces, which is much less labor-intensive than welding into a cylindrical or spherical chamber and therefore much cheaper. Furthermore, the chamber has to house the cooling shield, which must be rectangular because of a $\mathrm{X}-\mathrm{Y}-\mathrm{Z}$ table inside of it which is moving a penetrometer and other sensors above the sample. A cylindrical chamber would have to be bigger in order to house this cooling shield. The downside compared to a cylindrical or spherical chamber is that the force created by the pressure difference between the ambient pressure outside and the vacuum inside the chamber has to be withstood by the material, since the shape does not provide as much resistance as a cylindrical or spherical chamber would. In order to compensate for this, the wall thickness had to be between 20 and $30 \mathrm{~mm}$, depending on the number and size of flanges in each plate. The total weight of the empty chamber is $1100 \mathrm{~kg}$; when fully assembled, the weight is around $1700 \mathrm{~kg}$, depending on the specification chosen. Most of the surface plates were welded to the main chamber in order to reduce cost. Only the front and the top plates of the chamber are removable and can be moved using a crane. This is necessary for the installation of the cooling shield and other large 
interior structures. For smaller construction work inside the chamber and for the sample transfer, the chamber is equipped with two quick-release doors.

In order to accommodate all instruments, the L-Chamber has 50 flanges in total. This is to provide each instrument with its own flange for observational windows and electrical feedthroughs. This system allows for individual installation and operation of the different instruments, which provides high flexibility while planning different experiment campaigns. Figures $1-5$ show the positions of all flanges on the L-Chamber. Each flange is given a designated code consisting of a specific letter for each plate and a number. The assigned letter and the total number of flanges for each plate is shown in Table II. In Table III, the specific type and usage of each flange is presented. Note that some flanges are grouped together because they are built for the same purpose and are of the same type.

\section{B. Vacuum pumps}

For creating the vacuum in the L-Chamber, a combination of a pre-pump and a turbo-molecular pump (TMP) is used. For the prepump, we use a dry multi-stage roots pump. Using an oil-free pump is important because the oil from a rotary vane pump could contaminate the samples, which would affect the results from the mass spectrometer and the optical measurements. The maximum pumping rate is $37 \frac{\mathrm{m}^{3}}{\mathrm{~h}}$ so that the whole chamber can be evacuated within a few minutes. However, this will only be done if no sample is inside the chamber because the maximum pumping rate would most probably destroy the highly porous granular sample. In turn, this would damage the pumps and the samples would be lost. Therefore, the

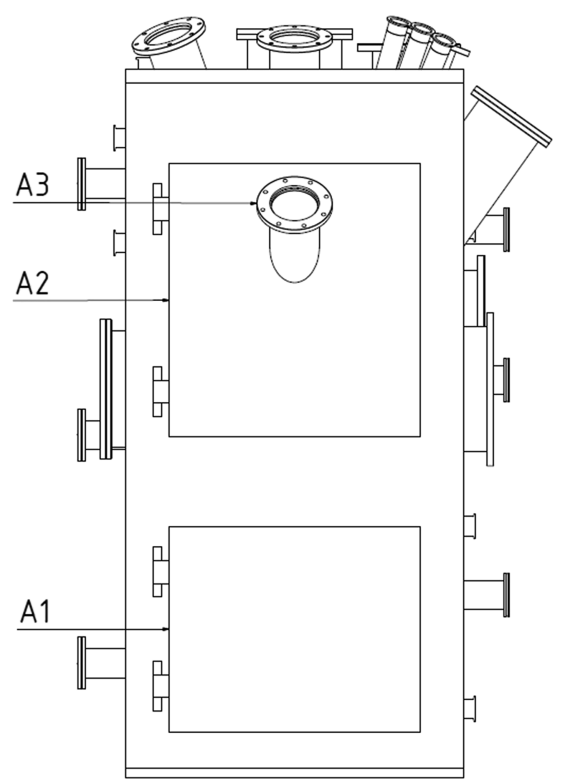

FIG. 1. In this drawing, the front plate of the L-Chamber is shown. The height of the chamber is $1640 \mathrm{~mm}$, and the width seen from this side is $1050 \mathrm{~mm}$. It has two quick-release doors and one flange integrated into the upper door. The whole plate can be removed from the chamber for transport or construction work. The flange type and assignment are given in Table III.

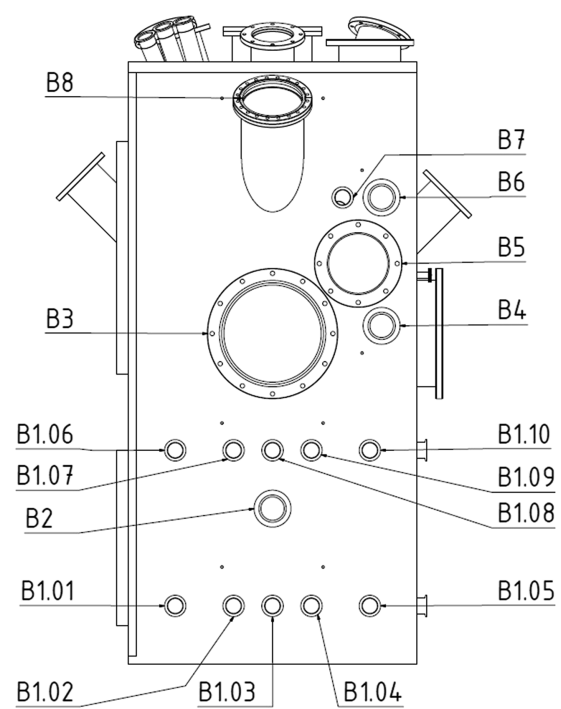

FIG. 2. In this drawing, the right plate of the L-Chamber is shown. The height of the chamber is $1640 \mathrm{~mm}$, and the width seen from this side is $1070 \mathrm{~mm}$. It houses a total of 17 flanges for viewports, valves, and feedthroughs. The flange type and assignment are given in Table III.

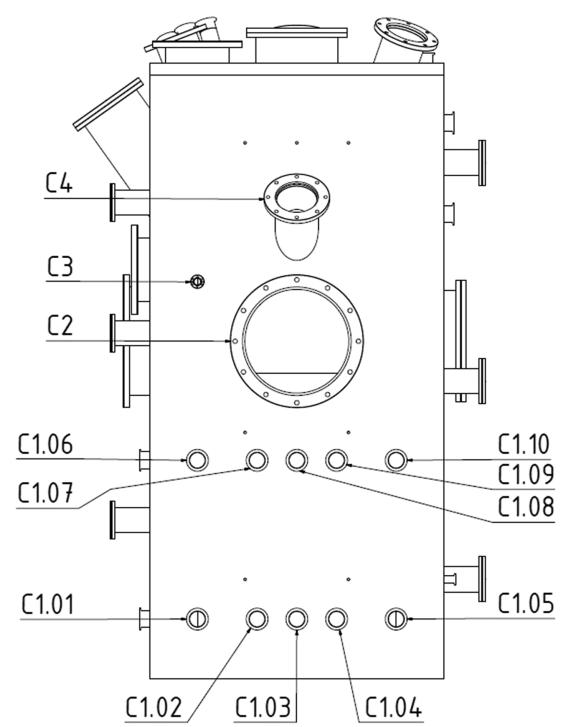

FIG. 3. In this drawing, the rear plate of the L-Chamber is shown. The height of the chamber is $1640 \mathrm{~mm}$, and the width seen from this side is $1050 \mathrm{~mm}$. It houses a total of 13 flanges for viewports, valves, and feedthroughs. Also the burst window and an over-pressure valve are located here. The flange type and assignment are given in Table III.

pumping rate must be controlled and carefully adjusted. To this end, we installed the setup described below and shown in Fig. 6 .

For safety reasons, the TMP is directly attached to the LChamber. Behind the TMP, an electronically controlled pneumatic valve is attached directly to the TMP. This valve is connected to a 


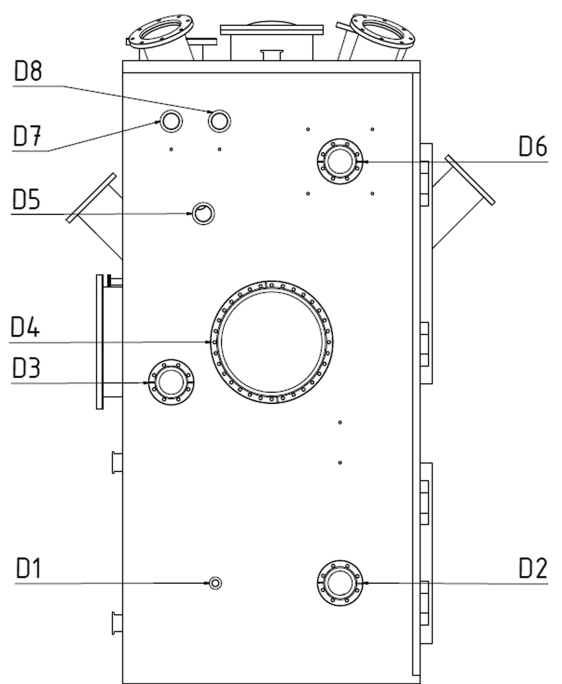

FIG. 4. In this drawing, the left plate of the L-Chamber is shown. The height of the chamber is $1640 \mathrm{~mm}$, and the width seen from this side is $1070 \mathrm{~mm}$. It houses a total of eight flanges, including the connection for the turbo-molecular pump and for the liquid-nitrogen supply. The flange type and assignment are given in Table III.

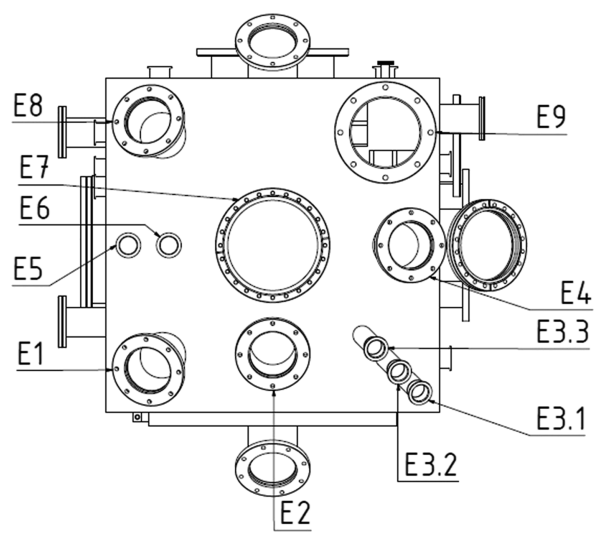

FIG. 5. In this drawing, the top plate of the L-Chamber is shown. Measured over the longest distances, the size of the chamber seen from top is $1050 \mathrm{~mm}$ times 1070 $\mathrm{mm}$. It houses a total of 11 flanges for viewports, feedthroughs, and liquid-nitrogen supply. The flange type and assignment are given in Table III.

T-cross, from which a flexible tube runs to the pre-pump, forming the $40 \mathrm{~mm}$ main pumping line. A second line runs from the T-cross directly to the chamber, forming a bypass to the TMP. Another electronically controlled pneumatic valve and an electronically controlled needle valve are installed in this bypass. At the beginning of evacuation, the line to the TMP is closed and only the bypass is open. The needle valve is used to control the pumping speed according to a pre-determined pumping-speed profile. This allows the evacuation without destroying the sample. For additional safety, the bypass line is equipped with a $4 \mu \mathrm{m}$ filter to stop dust reaching the pre-pump. Although not planned for the first experiments, this system allows also the simulation of low pressure environments, like on Mars or on Pluto. After reaching a pressure below $10^{-1}$ mbar
TABLE II. Summary of the different L-Chamber components and the total number of flanges.

\begin{tabular}{lcc}
\hline \hline Letter & Chamber plate & Number of flanges \\
\hline A & Front plate & One flange and two doors \\
B & Right sidewall & 17 \\
C & Rear plate & 13 \\
D & Left sidewall & 8 \\
E & Top plate & 11 \\
\hline \hline
\end{tabular}

inside the chamber, the bypass is closed and the main pumping line is opened. The TMP is then started with the aim to decrease the pressure in the chamber to the operational value around $10^{-6} \mathrm{mbar}$.

The two electronically controlled pneumatic valves are normally closed, which means that in the case of a power blackout, the pneumatic system isolates the pre-pump from the main chamber. In this case, the TMP is automatically stopped to prevent damage but is not disconnected from the main vacuum chamber, since it has to be mounted directly at the chamber wall for structural reasons. The pre-pump is protected by a fourth valve, which is normally open. This valve lets air in the pumping line between the pre-pump and the pneumatic valves. This safety valve is closed as soon as power is turned on and it is not actively controlled. The other valves are all controlled by micro-controllers, which receive commands from the control computer.

\section{Cooling shield}

The main task of the cooling shield, shown in Fig. 7, is to minimize the radiative heat input from the warm sidewalls of the L-Chamber. Furthermore, the structure of the shield has to hold MARA and the X-Y-Z table, which moves the sensors above the sample. The cooling shield also must allow the observation of the sample by the various instruments installed outside of the L-Chamber. Thus, it needs holes in all viewing axes. The size of the cooling shield is determined by the maximum sample height, which is $300 \mathrm{~mm}$. In addition to the sample height, the sample holder measures $120 \mathrm{~mm}$. Hence, the main opening in the cooling shield has to allow the transfer of a $420 \mathrm{~mm}$ high structure. In addition, the planned usage of a penetrometer requires enough space to store above the sample a needle with a length similar to the sample height. The resulting height of the cooling shield is $830 \mathrm{~mm}$ as measured on the front side.

The shape of the cooling shield is rectangular, which is required because of the $\mathrm{X}-\mathrm{Y}-\mathrm{Z}$ table installed inside. Due to its rectangular shape, the cooling shield consists of five cooling plates, which are mounted to a frame constructed from aluminum profiles. The front plate also functions as a door, which can slide down on rails in the frame to allow for the insertion of the sample.

For the active cooling, liquid nitrogen will be used, since the operating temperature of the plates should be at $90 \mathrm{~K}$. The size of the cooling plates renders a conventional system with copper tubes soldered to metal plates difficult because it would require steadily flowing nitrogen, which would lead to a very high nitrogen consumption. To allow for long experiment campaigns, the cooling shield and also the sample cooling system (see Sec. III D) have to be optimized for a low consumption of liquid nitrogen. 
TABLE III. Specification of each L-Chamber flange and its usage.

\begin{tabular}{|c|c|c|}
\hline Flange code & Flange specification & Flange assignment \\
\hline \multicolumn{3}{|r|}{ Front plate } \\
\hline A1 & Quick-release door $500 \times 400 \mathrm{~mm}^{2}$ & Cleaning of the chamber and small maintenance work \\
\hline $\mathrm{A} 2$ & Quick-release door $500 \times 550 \mathrm{~mm}^{2}$ & Sample transfer and small maintenance work \\
\hline $\mathrm{A} 3$ & ISO-F 100 & Optical window at a $45^{\circ}$ an angle for surface observation and illumination of cliffs \\
\hline \multicolumn{3}{|r|}{ Right sidewall } \\
\hline $\mathrm{B} 1.01-\mathrm{B} 1.10$ & ISO-KF 40 & Scale feedthrough, flooding valves, and pressure sensors \\
\hline B2 & ISO-K 63 & Feedthrough for scale lifting system \\
\hline B3 & ISO-F 250 & Window for high-speed cameras for 3D particle tracking \\
\hline B4 & ISO-K 63 & Feedthrough for heatstrip \\
\hline B5 & ISO-F 160 & Electrical feedthrough with 74 pins for PT1000 temperature sensors \\
\hline B6 & ISO-K 63 & MARA signal feedthrough \\
\hline B7 & ISO-KF 40 & MARA safety heater power supply \\
\hline B8 & ISO-CF 160 & Window for optical reflection measurements \\
\hline
\end{tabular}

\begin{tabular}{|c|c|c|}
\hline \multicolumn{3}{|r|}{ Back plate } \\
\hline $\mathrm{C} 1.01-\mathrm{C} 1.10$ & ISO-KF 40 & Power supply for scale heaters, thermocouple feedthroughs, and excess pressure valve \\
\hline $\mathrm{C} 2$ & ISO-F 250 & Window for high-speed cameras for 3D particle tracking \\
\hline $\mathrm{C} 3$ & ISO-CF 16 & Burst disk as a safety device in case of rapid pressure buildup due to ice evaporation \\
\hline $\mathrm{C} 4$ & ISO-F 100 & Optical window at a $45^{\circ}$ an angle for surface observation and illumination of cliffs \\
\hline \multicolumn{3}{|r|}{ Left sidewall } \\
\hline D1 & ISO-CF 16 & Bypass pumping line \\
\hline D2 & ISO-CF 63 & Nitrogen input for the sample cooling system \\
\hline D3 & ISO-CF 63 & Nitrogen output for the sample cooling system \\
\hline D4 & ISO-CF 250 & Reinforced flange for the turbo-molecular pump \\
\hline D5 & ISO-KF 40 & Power supply for the $\mathrm{X}-\mathrm{Y}-\mathrm{Z}$ table \\
\hline D6 & ISO-CF 63 & Feedthrough for four glass fibers for the illumination system \\
\hline D7 & ISO-KF 40 & Feedthrough for penetrometer data \\
\hline D8 & ISO-KF 40 & Power supply for cooling shield doors \\
\hline
\end{tabular}

\begin{tabular}{ccc}
\hline & & Top plate \\
\hline E1 & ISO-F 100 & Optical window for surface observation, high-speed cameras, or light shield \\
E2 & ISO-F 100 & Zinc-selenide window for the infrared camera \\
E3.1 - E3.3 & ISO-KF 40 & Angled flanges with equal distance for the mass spectrometer \\
E4 & ISO-F 100 & Synthetic air inlet \\
E5 & ISO-KF 40 & Pressure sensor \\
E6 & ISO-KF 40 & Fused silica window for solar simulator \\
E7 & ISO-CF 200 & Opindow for surface observation, high-speed cameras, or light shield \\
E8 & ISO-F 100 & Nitrogen inlet and outlet for the cooling shield \\
E9 & ISO-F 160 & Optight shield \\
\hline \hline
\end{tabular}

In our case, the so-called pillow plates were installed inside the L-Chamber. These plates are made from two stainless-steel plates, which are welded together along the edges and at specified points. During construction, water at high pressure is forced between the plates to expand the plates, which creates a volume between them. Due to the welding pattern, the resulting shape resembles a pillow, hence the name. After drying out the water, the inner volume of the pillow plates can be filled with liquid nitrogen to evenly cool the plates. The main advantages of this system are, first, that a standing liquid-nitrogen reservoir can be used, which provides a maximum cooling rate while minimizing the liquid nitrogen consumption. In a system made out of tubes welded to a plate, this would not be possible because the back pressure in the tubes would not allow the liquid nitrogen to fill the system without active pumping. Second, these plates also allow the implementation of viewing ports at all required positions with minimal disturbance to the temperature field.

The liquid nitrogen that is required to cool the cooling shield is supplied from a reservoir mounted above the chamber. Gravity forces the liquid nitrogen into the plates. This reduces the 


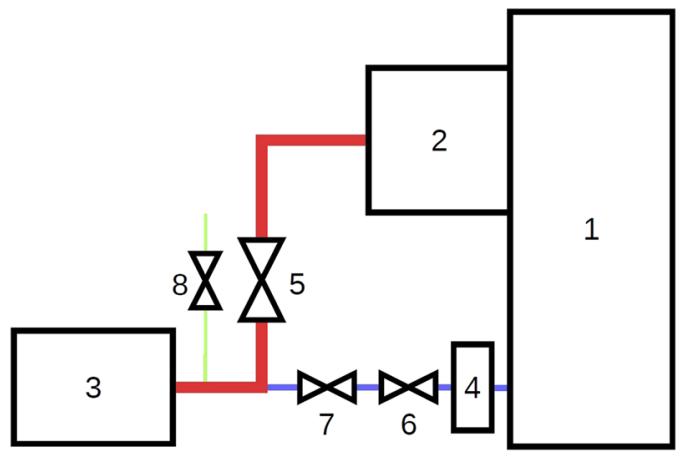

FIG. 6. Schematic drawing of the pump system used for the comet-simulation chamber. (1) L-Chamber, (2) TMP, (3) oil-free pre-pump, (4) $4 \mu \mathrm{m}$ filter, (5) 40 $\mathrm{mm}$ electronically controlled pneumatic valve, (6) $16 \mathrm{~mm}$ electronically controlled pneumatic valve, (7) needle valve, and (8) emergency flooding valve. The bold red line is the main pumping line, the blue line marks the bypass line, and the green line is the emergency flooding line.

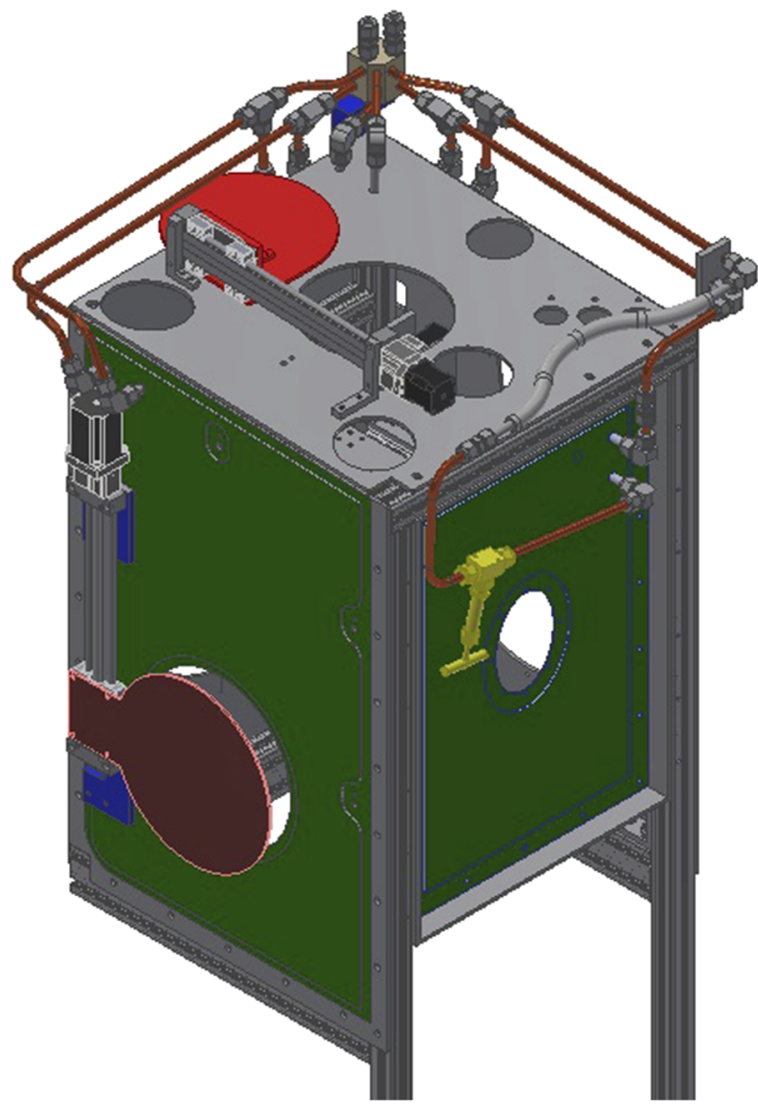

FIG. 7. 3D model of the cooling shield viewed from a $45^{\circ}$ angle. The nitrogen distributor made from brass is mounted at the top right corner. The nitrogen flows in through the left tube and the gas leaves the system through the right tube toward the exhaust. The nitrogen line feeding the door has an integrated nitrogen valve (yellow) that is closed before the door is disconnected from the pipe. Above the top plate, a moving plate is attached (bright red plate) that can shield the sample from the warm window of the solar simulator. The second moving plate (dark red), mounted on the left cooling plate and used to protect the TMP against dust, is closed. operational effort because only the filling height of the reservoir must be controlled. This is done by a magnetic valve, which is operated by a micro controller. The filling height is measured by temperature sensors inside the reservoir such that it is kept between two fixed heights. The valve is normally closed. Hence, in the event of a power blackout, the liquid-nitrogen line is closed, which would result in a slow warm-up of the cooling shield in case of emergency. The weight of the plates is around $65 \mathrm{~kg}$, which means that the heat capacity is very high, and even after the nitrogen has evaporated, the L-Chamber can be kept cold for an extended time period and the sample is not altered if power can be reinstalled in a few hours.

The cooling shield has holes for all optical experiments and also toward the TMP for high pumping efficiency. However, these holes are heat leaks, which decrease the cooling efficiency. In particular, the holes for the high-speed cameras and the reflectance measurements have a big impact due to their size. In order to minimize the heat loses, the biggest holes can be covered by adaptable covers. These are made from aluminum and cooled due to the contact to the cooling shield plates. By using the covers, the holes can be reduced in size or completely covered if they are not in use. In addition to the fixed covers, there are two moving plates attached to the cooling shield, which cover the holes for the solar simulator and for the TMP. The hole toward the TMP is only uncovered after a pressure below $10^{-4}$ mbar is reached and it protects the TMP against dust, which could be lifted during the evacuation process. This hole can be also closed if high activity of the sample is detected. The plate toward the solar simulator is used to simulate cold nights because it can shield the sample toward the window of the solar simulator. Even if the solar simulator is turned off, the window is still warm, and the resulting radiation would influence the sample. Both plates are mounted to a rail system and powered by a stepper motor, so they can be placed in any position along the rail, for example, to create a sharp shadow on the sample.

All surfaces of the cooling shield facing the sample are painted black by using NEXTEL-Velvet-Coating 811-21 from the company Mankiewicz Gebr. and Co. (GmbH and Co. KG). This painting has an infrared emissivity of 0.99 ; hence, it maximizes the heat transfer by radiation. The same paint is used on the sample holder and on the sample cooling system (see Sec. III D).

\section{Sample cooling system}

The purpose of the sample cooling system, shown in Fig. 8, is to cool the sample holder and thereby the sample from below to simulate the relative low and constant temperature beyond the skin depth of a comet. In addition, the sample cooling system is designed around the use of the scale inside the L-Chamber, which introduces some additional requirements. First, the scale has to be sufficiently far away from the cold parts because it cannot be operated low temperatures. Second, the cooling system cannot be in contact with the scale to not disturb the weight measurement. We decided to design a cooling system that cools by radiation only, and thus, large surface areas are needed. The core of this system is the donut-shaped liquidnitrogen tank directly below the sample holder, which is connected to a liquid-nitrogen tank located outside the chamber. Both tanks 


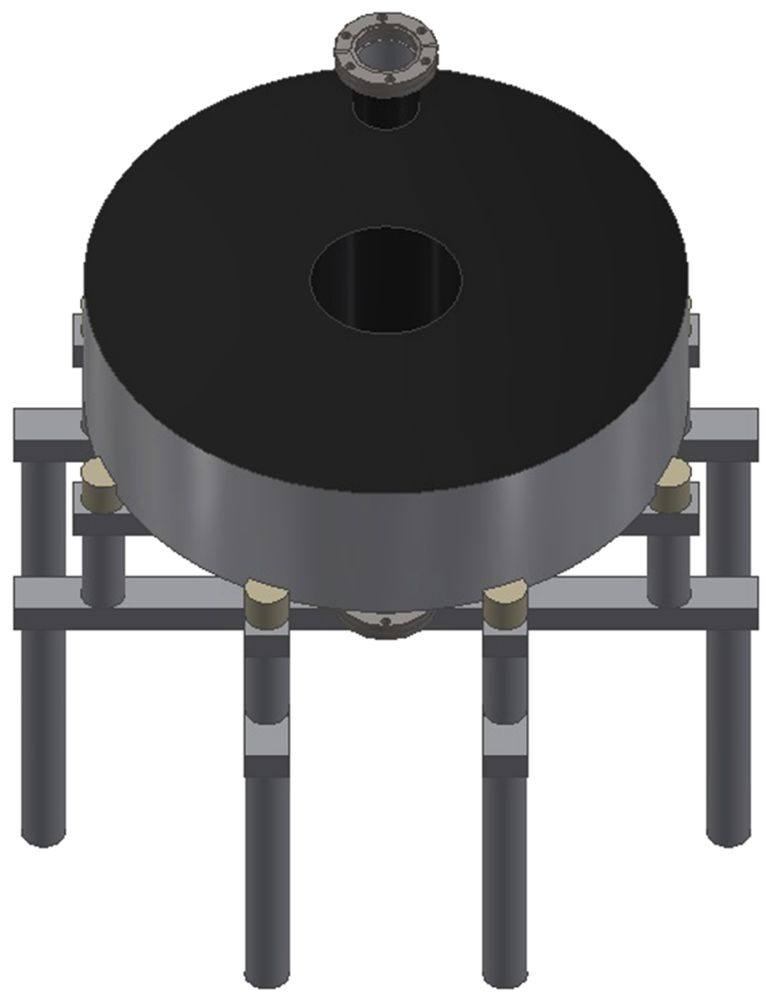

FIG. 8. 3D model of the sample cooling system placed on its holding structure viewed from a $45^{\circ}$ angle. The donut is part of a u-shaped tube, and hence, it will always be filled with liquid nitrogen. Copper sticks (not visible here) are installed inside the donut-shaped liquid nitrogen tank to increase the heat transfer from the liquid nitrogen to the top plate of the donut. The top plate and the wall of the hole are painted black to increase the radiative heat transfer from the tank to the sample holder. The donut is placed on eight TECAPEEK spacers (beige) to reduce the heat loss to the aluminum structure below.

are connected forming a u-shaped tube. By controlling the filling height of the tank outside, it can be ensured that the donut is always completely filled with liquid nitrogen. The filling height is also monitored by temperature sensors inside the donut. To further optimize the cooling performance, a total of ten copper rods with a diameter of $18 \mathrm{~mm}$ are attached to the top of the donut reaching into the liquid nitrogen. These heat pipes ensure a good heat transfer from the liquid nitrogen to the top plate of the donut, even if gas pockets form temporarily inside the donut.

The sample holder consists of a copper plate with $400 \mathrm{~mm}$ diameter, which is attached to a copper tube with $100 \mathrm{~mm}$ diameter and $120 \mathrm{~mm}$ in length. This tube fits through the hole in the middle of the donut with $1 \mathrm{~mm}$ clearance around it. The bottom of the copper tube is connected to the scale via a lightweight TECAPEEK construction, consisting of four tubes with diameters of $10 \mathrm{~mm}$. This minimizes the heat flow from the cold parts to the scale at the bottom of the L-Chamber. While the scale is in use, the copper plate is resting $2 \mathrm{~mm}$ above the surface of the donut. The radiative heat transfer from the donut to the sample holder is increased by using the same black paint as on the cooling-shield plates.

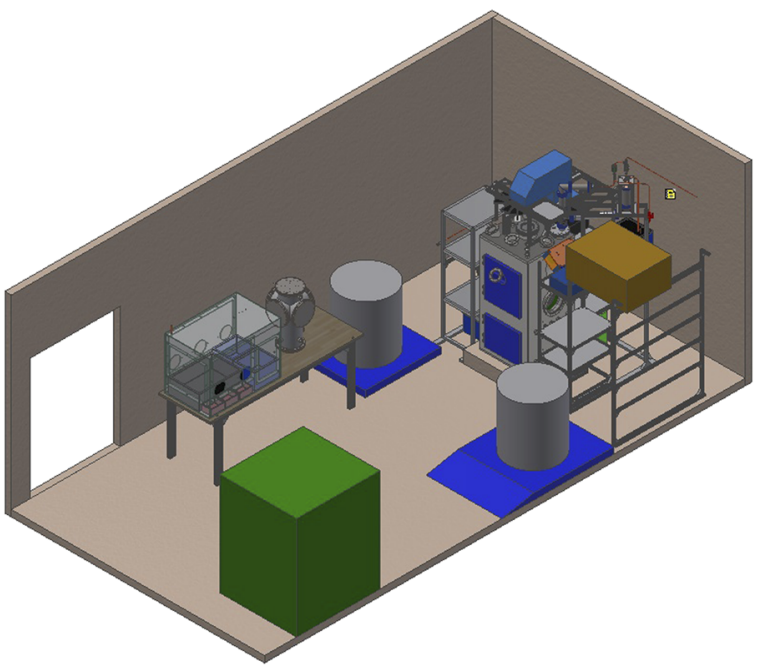

FIG. 9. Sketch of the CoPhyLab laboratory room seen from a $45^{\circ}$ angle. The chamber is located at the rear part of the room (top right in the sketch) and is surrounded by the experiment towers. Left and right in the front of the chamber, the liquid-nitrogen tanks are placed on floor scales. In the front left corner of the room is the sample-preparation table with the glove-box (Sec. V A) and the ice-drying chamber. The S-Chamber (dark green), which is used for smaller experiments, is placed in the front right corner of the room.

\section{E. Surrounding infrastructure}

The L-Chamber is surrounded by three experiment towers (see Fig. 9), which contain the cameras, control electronics, and power supplies that are required for all instrument and chamber operations. In addition, the solar simulator rests on one of these towers. The towers are constructed from aluminum profiles and plates so that the height of each plate is freely adjustable. The plates that hold the high-speed cameras are reinforced to allow for high precision positioning of the cameras without bending of the plates. Data and power supply cables connect the equipment with the control computer in a neighboring room. We decided to use a control room for the L-Chamber to minimize safety issues and installed a ventilation system and an oxygen sensor to secure safe working conditions in the experiment room. An additional safety measure is that the whole chamber, e.g., its valves, motors, and pumps, is operated by micro-controllers since they are more reliable than computers in long-duration experiments. The system is monitored by a safety controller, which has no connection to the main control computer. This controller monitors the temperatures, the pressure, and the state of all valves, pumps, and motors. In case of malfunction, the safety controller can automatically take action to secure a safe operational state. Furthermore, a backup power supply is installed to provide power to all valves, micro-controllers, and the computer during a power blackout. This ensures that in the event of a blackout, only the pumps and the solar simulator are turned off, but the chamber remains in a secure state.

For the preparation of the sample, a custom-made glove-box (see Sec. V A for details) is used. From there, the samples are transported by a specially designed sample-transport cart, which is presented in Sec. V B. 


\section{F. Solar simulator}

For the illumination of the samples, a solar simulator (Solar Simulator LS1600 from Solar Light Company) is used. The beam diameter is $160 \mathrm{~mm}$, and the area can be illuminated with up to 1.3 solar constants. The solar simulator is mounted to the rear experiment tower and is positioned above the chamber so that the incidence direction is vertically downward. The power supply of the solar simulator is also placed on the rear tower, and both units are cooled by fans to avoid overheating. The light beam passes through a window procured by Pfeiffer Vacuum Components and Solutions $\mathrm{GmbH}$ with a diameter of $198 \mathrm{~mm}$. This size is sufficient to avoid reflections on the window frame. The window material is fused silica, so it is transparent to the whole solar spectrum including UV. The UV light is not of particular interest for the currently planned experiments. However, this choice allows us to study the influence of the UV part of the spectrum on comet activity at a later stage of the project. The total distance between the lens of the solar simulator and the sample surface is $1208 \mathrm{~mm}$. The power input of the solar simulator at the sample surface was measured in the final setup at maximum output power; the result is shown in Fig. 10.

For the simulation of cometary day-night cycles, the solar simulator is accompanied by three additional devices, namely, a shutter, a movable door on the cooling shield, and a mechanical dimming device. The shutter is mounted inside the solar simulator housing and can be used to interrupt the beam before it leaves the solar simulator. This is required because turning the lamp frequently on and off shortens its lifetime dramatically. In the normal operation mode, the lamp will be kept running for the whole experiment time, which can last up to several weeks. The movable door was already introduced in Sec. III C and is used to ensure cold nights when the cold door blocks the light path. In this case, the hot parts of the solar simulator and the warm window cannot heat the sample surface anymore. To enable a smooth transition between the day and the night case, a dimming device was developed (see Fig. 11), which is also positioned in the light path underneath the lens of the solar simulator but outside of the chamber (see Fig. 12). It consists of eight elements stacked

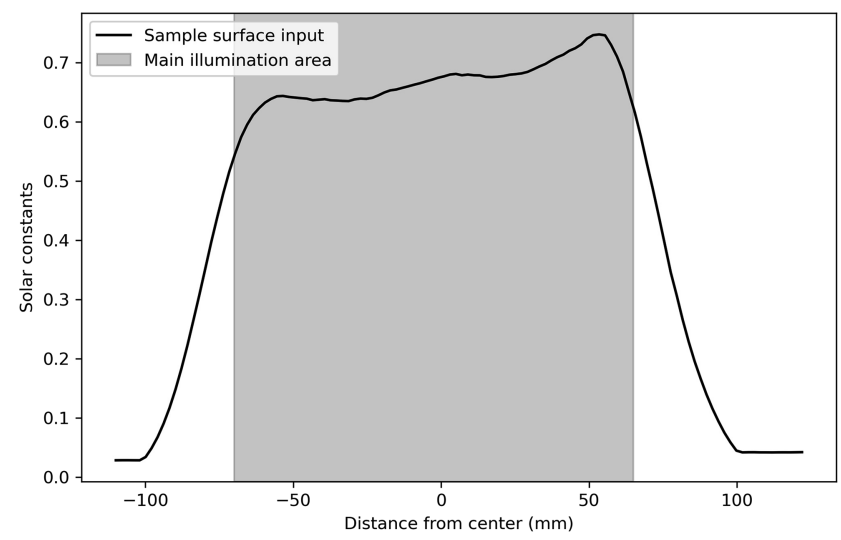

FIG. 10. Measured light intensity profile in the plane of the sample surface. The measurement was taken along the center line of the sample, and 0 indicates the center of the sample. The maximum intensity is $0.72 \mathrm{E}_{0}$. The visible gradient will be improved by a new solar simulator in the future.
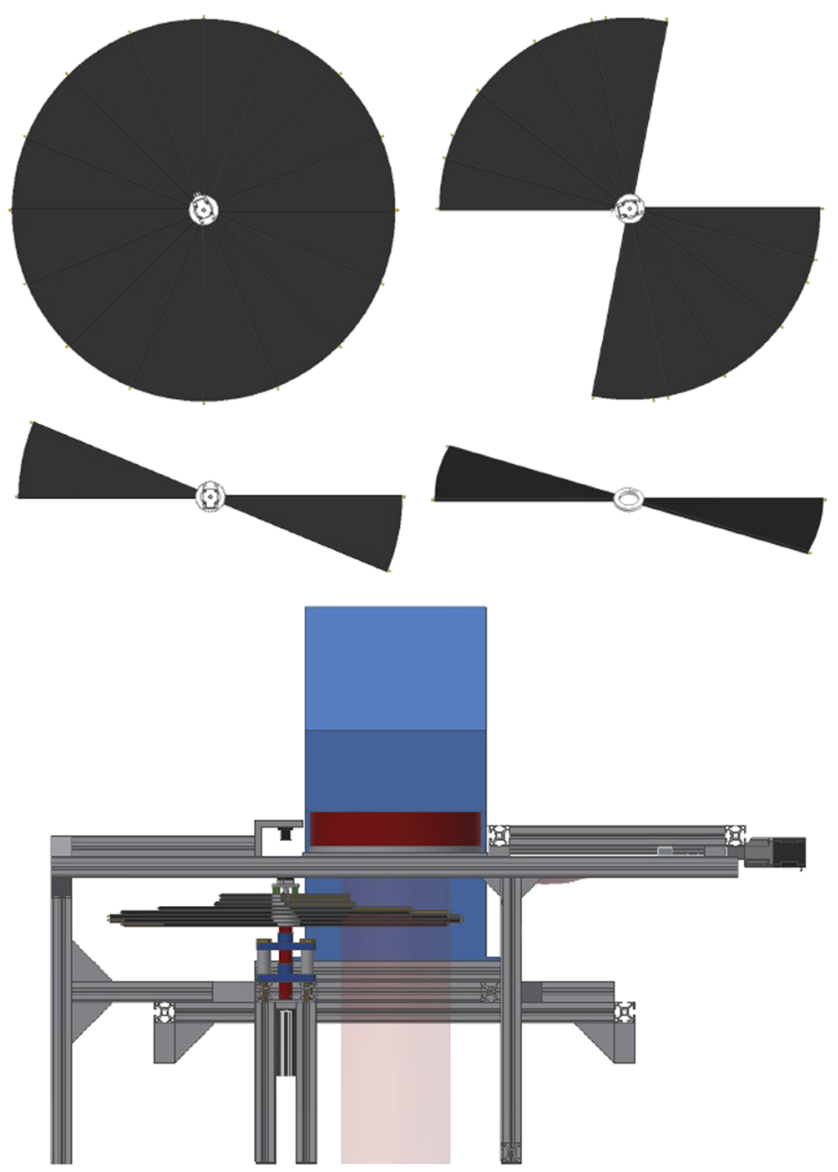

FIG. 11. Mechanical dimming device in three positions: fully closed (top left), half open (top right), and open to maximum (center left). Also a single element is shown (center right). The bottom part shows the installation of the dimming device underneath the solar simulator viewed from the front. It is mounted on the left side of the solar simulator, and the actuator motor is mounted under the aluminum profile holding the chopper. The light beam of the solar simulator is shown in light red going straight down with a diameter of $160 \mathrm{~mm}$.

on top of each other over a central axis and a stepper motor (dimmer motor) mounted on top of this axis. The lowest element is fixed to the central axis, while the highest element is connected to the dimmer motor. Each element has two wings mounted on opposite sides of the central element. Each wing is $240 \mathrm{~mm}$ long and made of two aluminum spokes spaced at a $22.5^{\circ}$ and a $3 \mathrm{D}$-printed flap, which is fixed to the spokes on both long ends. Hence, both wings together cover $45^{\circ}$. All elements are interlocked so that they can only turn by $45^{\circ}$ relative to the elements next to them. If all eight elements are stacked directly above each other, they cover a total of $45^{\circ}$. By using the dimmer motor, the covered angle can be increased from $45^{\circ}$ to the full $360^{\circ}$. The whole dimming device including the dimmer motor is turned at a constant speed by using a second stepper motor (actuator motor) connected to the central axis. Hence, over one revolution, the reduction in power can be between $12.5 \%$ and $100 \%$. This enables us to replicate sunrise and sunset on a comet. 


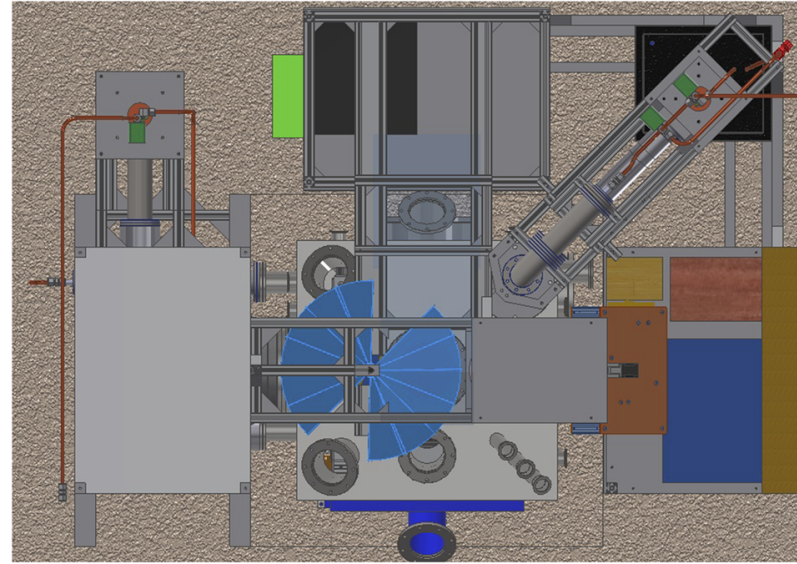

FIG. 12. Schematic view of the chamber from above. The mechanical dimming device, which is placed between the solar simulator (grayed out in this picture) and the window of the chamber, is highlighted in blue color. The motor turning the device is mounted underneath the chopper. The structure is attached to the rear and left experiment towers by aluminum profiles.

\section{INTEGRATED INSTRUMENTS}

\section{A. Mechanical properties}

\section{Penetrometer (standard variant)}

During an experiment run, it is expected that the texture of the sample changes due to the input of thermal energy and internal sublimation processes. Gas that is set free inside the sample material can escape from the sample or re-condense in the interior of the sample. This process can lead to strong changes in the thermal and mechanical properties of the sample material. To investigate such effects, we installed a movable penetrometer needle inside the L-Chamber. This device is capable of measuring the compressive strength of the sample before during and after an experiment run (see Fig. 13).

The penetrometer is mounted on a platform inside the cooling shield. This platform can be moved in the X-Y plane above the sample by a motor-driven rail-and-gantry system, which is mounted to the aluminum frame of the cooling shield. Once the penetrometer is in the desired position, another rail system, the Z-stage, can push the penetrometer needle into the sample. The needle is equipped with a load cell, monitoring the penetration force during the insertion process. ${ }^{54}$ A set of three different load cells of the type Entran ELFM with nominal load ranges of 25,100 , and $500 \mathrm{~N}$ are available for the tests. The standard needle has a total length of $120 \mathrm{~mm}$, and a diameter of $3.5 \mathrm{~mm}$ and is also equipped with temperature sensors in the tip, center, and rear part of the needle. Furthermore, it also can be actively heated, and therefore, it can be used as a line heat thermal conductivity sensor as described in Sec. IV B 5. If samples with a height of more than $100 \mathrm{~mm}$ are used, the needle can be exchanged for a longer version without a heater and temperature sensors.

\section{Penetrometer (Brazilian disk test)}

A second application for the motor-driven rail-and-gantry system is a setup for measuring the tensile strength (see Fig. 14). In this case, the needle is replaced by a piston with a horizontal bar at the end. This bar is $30 \mathrm{~mm}$ long and $2 \mathrm{~mm}$ thick. The samples for this

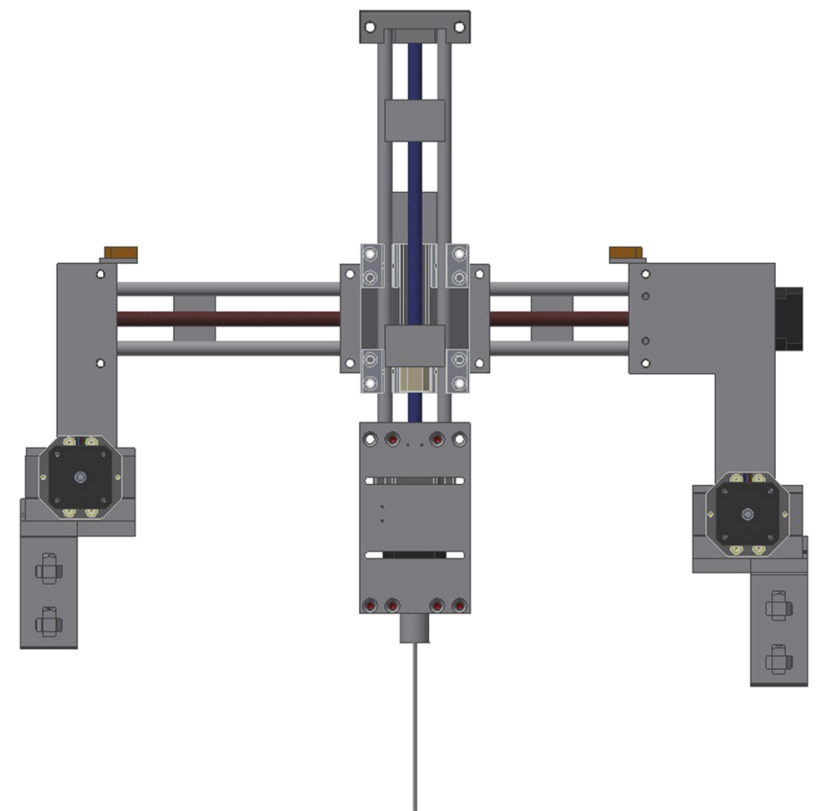

FIG. 13. 3D model of the standard penetrometer viewed from the front. It is constructed out of four stepper-motor-driven rails and is mounted to the aluminum frame of the cooling shield. The two stepper motors on the left and right side are used to drive the penetrometer back and forth along the $\mathrm{Y}$-stages. The $\mathrm{X}$-stage is mounted horizontally between the $Y$-stages. A third stepper motor is used to drive the penetrometer from side to side. Finally, the Z-stage is mounted to the $\mathrm{X}$-stage. It is driven up and down using a fourth stepper motor. Underneath the Z-stage, a force cell is installed to which the penetrometer needle is attached.

measurement are disks with a diameter of $25 \mathrm{~mm}$ and a length of $20 \mathrm{~mm}$. This setup can be used to perform the Brazilian disk test. By this method, a disk is compressed by the piston and the exerted pressure is measured by the load cell. The force needed for obliteration is used to calculate the tensile strength of the sample. ${ }^{20,55}$ The main

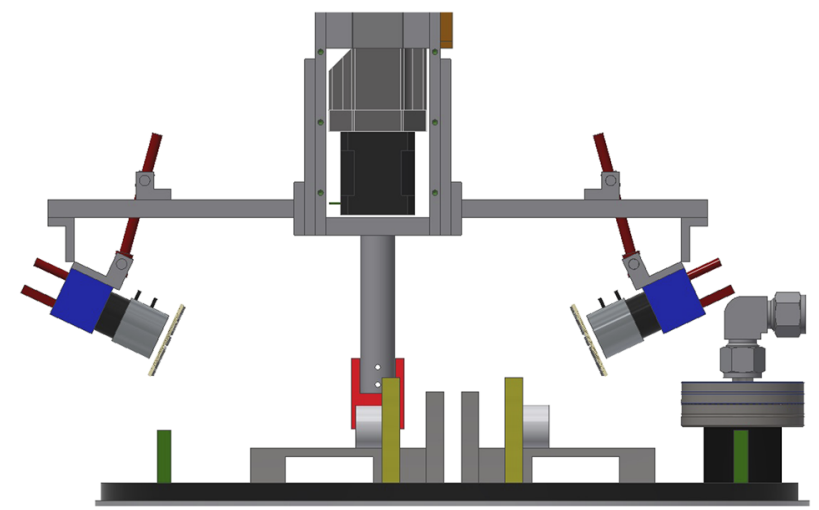

FIG. 14. 3D drawing of the Brazilian disk test setup inside the cooling shield Eight identical samples can be placed on the sample holder (black bottom plate). The penetrometer is equipped with a $30 \mathrm{~mm}$ long and $2 \mathrm{~mm}$ thick piston (bright red). The crack in the sample is observed by the front camera (here on the left side), which is attached to the Z-stage of the penetrometer. Both cameras form the mobile stereo imaging system detailed in Sec. IV C 2. 
advantage of this design is that the test can be conducted inside the vacuum chamber. For one experiment run, eight identical disks can be placed on one sample holder inside the chamber. Four of them are placed in the illuminated area of the solar simulator and can be heated. The other samples are always in the shadow and therefore will experience less alteration. These four samples in the shadow can be used as reference samples if required.

It is possible to measure samples in different states. For example, we can produce samples with the same ice-to-dust ratio. The Brazilian disk test is then performed after different time periods so that the altering through sublimation can be tracked. To monitor the fracturing process, the samples are observed by the stereo imaging system (see Sec. IV C 2), which is attached to the Z-stage and moves along with the piston.

\section{B. Temperature}

For a complete physical description of the sample behavior under insolation, knowledge about the temperature distribution across the sample volume is mandatory. In this section, we describe how we determine the relevant temperatures in the L-Chamber.

\section{Infrared camera}

For the inspection of the temperature distribution on the sample surface, a non-cooled commercial infrared (IR) camera (type FLIR P50) will be used. The camera is not suitable for operation in vacuum; therefore, it is mounted outside the L-Chamber. The IR camera observes the sample surface through a zinc selenide ( $\mathrm{ZnSe}$ ) window with $60 \mathrm{~mm}$ diameter that is equipped with an anti-reflection coating. The anti-reflection coating reduces the flux of photons emitted by the infrared camera itself. The window is mounted to the ISO-F 100 flange attached to port E2 and was procured from Hositrad.

The IR camera is equipped with an $\frac{f}{0.7}$ lens and has a focal length of $36 \mathrm{~mm}$ and a field-of-view of $22.6^{\circ} \times 17.1^{\circ}$. This field-ofview results in an imaged area of about $320 \times 240 \mathrm{~mm}^{2}$ at a distance of about $860 \mathrm{~mm}$ between the lens and the sample surface. This means that nearly the whole sample can be observed with a pixel scale of $1 \mathrm{~mm}$. The noise-equivalent temperature difference of the camera is specified as $0.1 \mathrm{~K}$ at $300 \mathrm{~K}$ object temperature, which translates into $\sim 0.23 \mathrm{~K}$ at the formally lowest object temperature of $230 \mathrm{~K}$ (given by the manufacturer) and about $0.5 \mathrm{~K}$ at $200 \mathrm{~K}$. It should be emphasized that these values are a measure of the temperature differences that can be temporally and spatially resolved by the camera, and the absolute uncertainty of the IR camera is considerably higher, $\sim \pm 2 \mathrm{~K}$ for an object temperature ranging from 273 to $373 \mathrm{~K}$. In any case, the necessary extension of the lower object temperature range and the presence of the $\mathrm{ZnSe}$ window in the beam path require a re-calibration of the camera under these conditions. For this purpose, a circular blackbody will be placed instead of the sample inside the chamber and will be cooled by the sample cooling system and heated by built-in foil heaters. With this method, an in situ calibration target with different, but known, temperatures will be available for the calibration.

\section{MARA}

In addition to the IR camera, a flight spare of the MAscot RAdiometer (MARA) will be used to measure the surface temperature. MARA was successfully operated on the surface of the asteroid Ryugu as part of the payload of the DLR Mascot Lander of JAXA's HayaBusa-2 mission. ${ }^{56}$ Designed for operation in space, MARA can operate in vacuum and in a low temperature environment. The latter is possible due to internal heaters, which stabilize the operational temperature at the intended temperature set-point. This set-point is usually chosen between 220 and $300 \mathrm{~K}$. The MARA sensor head is placed inside the L-Chamber and observes the sample from a distance of about $225 \mathrm{~mm}$ at an angle of $35.3^{\circ}$ relative to the sample surface. The sensor head is mounted to an aluminum holding structure attached to the cooling-shield frame. It is located in the rear right corner of the cooling shield at $45^{\circ}$ relative to the $\mathrm{x}$ and $y$ axis. In that position, it is as close to the sample center as possible without being directly above the sample, which would make the sample transfer impossible. Furthermore, MARA does not obscure any optical measurements in this position because it is located under the nitrogen-supply lines for the cooling shield. Directly above the MARA sensor head, an aluminum plate is mounted, which covers the sensor as seen from above. This plate protects the sensor head from mechanical forces, should the penetrometer experience a malfunction. MARA consists of several thermopiles, and the sensor voltages are transmitted as analog signals via a feedthrough to the electronics located outside of the L-Chamber.

MARA provides unresolved measurements of the emitted flux from a spot of the sample surface in six infrared wavelength channels between 6 and $50 \mu \mathrm{m} .{ }^{57}$ Due to the tilted orientation of the sensor head, the observed spot on the sample's surface is an ellipse, which covers nearly the whole illuminated part of the sample in one direction and about half of that distance in the perpendicular direction. In the two broadband channels, the absolute $2 \sigma$ uncertainty of the brightness temperature is about $1.5 \mathrm{~K}$ at an object temperature of $200 \mathrm{~K}$, which is considerably better than the absolute measurement capability of the IR camera at these low temperatures. Therefore, the MARA measurements can be used as tie points for the IR camera. This provides a considerable reduction of the final measurement uncertainty in the infrared images. The measurements will be complemented by using the scale and the mass spectrometer on pure water-ice samples, which can provide the temperature of the sample surface by calculating the sublimation rate (this technique is explained in Sec. IV D 2).

\section{PT1000 temperature sensors}

One of the methods used to measure the internal temperatures of the CoPhyLab samples is to place a set of PT1000 sensors (P1K0.232.3FW.A.007 from RS electronics) with a temperature range from 73 to $573 \mathrm{~K}$ and a long-term stability of less than $0.04 \%$ over $1000 \mathrm{~h}$ inside the volume of the sample. These sensors will measure the vertical and radial heat propagation inside the sample.

A total of 26 sensors will be used and their positioning is illustrated in Fig. 15. Eight sensors are placed in the central vertical line of the sample at depths of 5,10,16, 25, 40,55, 70, and $100 \mathrm{~mm}$, respectively. A higher spatial resolution near the surface is foreseen because we expect that the temperature changes will be strongest in the first few centimeters. Nine additional sensors are placed at a radial distance of $70 \mathrm{~mm}$ from the center. Stacked by three in the vertical direction, each triple sensor is offset by $120^{\circ}$ from their neighbors (see Fig. 15). The objective of these radial sensors is to detect horizontal deviations of the temperature distribution. The three stacked sensors are at a similar depth as the topmost central sensors $(5,10$, 


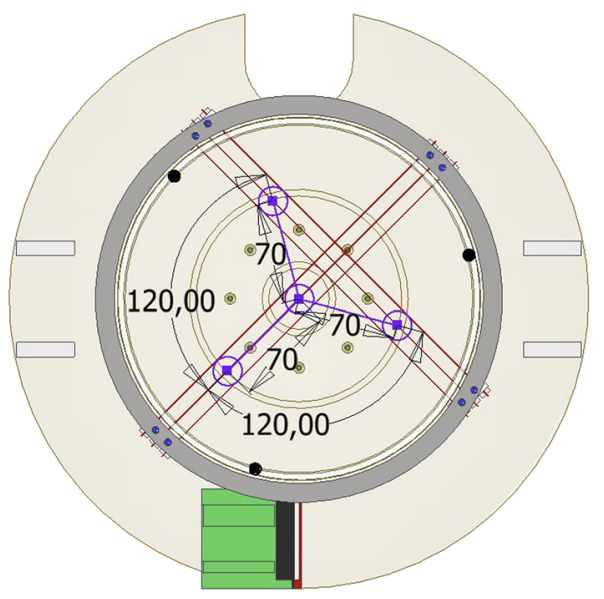

FIG. 15. Sketch of the position of the PT1000 sensors inside the sample. Eight sensors are located under the central blue circle at depths of $5,10,16,25,40,55$, 70 , and $100 \mathrm{~mm}$. These are mounted to the strings (red lines) spanned between aluminum posts screwed to the copper plate of the sample holder. They are not located directly above each other because the sensors would be to close together and the thermal contact to the sample would be compromised. Nine further sensors are located in the areas marked by the other three blue circles. They are located at a radius of $70 \mathrm{~mm}$ off-center and at a $120^{\circ}$ angle from each other. Below each circle, three sensors are placed at depths of 5,10 , and $16 \mathrm{~mm}$ spaced out for the same reason as the central sensors. An additional nine sensors are located at the sample holder wall below the black circles at depths of 10,30 , and $57 \mathrm{~mm}$. These sensors are also horizontally spaced by $120^{\circ}$. All cables from the sensors are connected to the main connector at the front of the sample holder, shown in green. After the sample holder is placed in the chamber, the harness is attached here before the chamber is closed.

and $16 \mathrm{~mm}$ ). Finally, nine sensors will be placed at the wall of the sample container to provide measurements of the boundary temperature conditions, which is required for the thermal models. The depths of these sensors are 10,30, and $57 \mathrm{~mm}$, respectively.

The total number of sensors present in the sample is limited by the number of cables that can exit the chamber, since each sensor has two cables attached to it. Furthermore, the thermal flow through the wires, which scales with their diameters, has to be minimized. The sensors are connected to a data logger through constantan wires to further reduce the heat transfer through the cables. The sensors are also attached to nylon strings installed between the walls of the sample container. The nylon strings were tested and found to be resistant to prolonged exposure to liquid nitrogen, and they have a low thermal conductivity. Furthermore, the nylon strings are thin and transparent, which reduces their influence on the CoPhyLab experiments.

\section{Heatstrip}

Different stationary and transient methods exist to determine the thermal conductivity, but only transient methods are applicable in the CoPhyLab experiments. Among these, the transient hot strip (THS) method is the most suitable to accurately determine the bulk thermal conductivity of the CoPhyLab samples. ${ }^{58}$ This method consists of placing a thin heater of large length-to-width ratio within the sample and then applying a constant amount of heating power.
Dependent on the thermal properties of the surrounding medium, the temperature of the strip will change, resulting in a characteristic self-heating curve. During the first part of the self-heating phase, the temperature increase is influenced by the thermal properties of the sample, the medium, and the contact resistance between the sample and the medium. However, for longer times, an asymptotic value will be reached, which solely depends on the thermal conductivity of the medium. In this asymptotic region, the thermal conductivity can be easily deduced from the inverse slope of the temperature rise when plotted against logarithmic time.

For the CoPhyLab L-Chamber, the THS is the heater of 70 $\mathrm{mm}$ length and $2 \mathrm{~mm}$ width mounted in a rigid frame structure made from a composite material used to stabilize the configuration. The heater wire is made of etched copper tracks of $150 \mu \mathrm{m}$ width that cover the whole strip in small meanders and provide a total resistance of about $8.5 \Omega$. By using copper as a resistance material, the heater can simultaneously be used as a temperature sensor due to the high temperature coefficient of the resistance of copper.

This special construction of the THS represents a reasonable approximation of an ideal THS sensor, which is characterized by "infinite" length and vanishing heat capacity. The latter is especially important when investigating very-low-density samples typical for comets. In this case, the finite heat capacity of, for example, typical needle probes, such as "line heat source" or "transient hot wire" probes, may not be negligible and could lead to significant deviations of the temperature curve from the ideal case and, thus, misinterpretations of the measurements. Another advantage of this technique is that always a finite area will be in direct contact with the medium, which reduces the problems caused by high contact resistance in granular media.

\section{Thermoneedles}

The probing tip of the penetrometer is a custom-made thermoneedle, making it a double-purpose instrument. Mechanically, the penetrometer is a stainless-steel tube with a diameter of $3.5 \mathrm{~mm}$ and a usable length of $80-100 \mathrm{~mm}$, depending on the version. The tip is a cone with a $60^{\circ}$ opening angle, which is a standard for penetrometer probes. A heater is positioned inside the needle, which can be used to warm up the whole probe. With three PT1000 temperature sensors, the probe temperature can be monitored at the top, middle, and bottom part of the tube. The heater wire inside the tube can be used to heat up the whole length of the probe, and together with the temperature sensors, the thermal conductivity of the samples can be investigated with this active sensing method. Due to the fact that the length-to-diameter ratio of the thermoneedle is between 22 and 28, a standard heat flow probe method cannot be applied. This would require the ratio to be at least 60 . A method for interpreting the measurements of these thermoneedles is described in the work of Tiefenbacher et al. ${ }^{59}$ The THS provides a more precise measurement, but in contrast, the needle can measure vertically and at different positions. In combination, both instruments will provide a very good overview of the thermal conductivity of the sample. In addition, the THS will be used for calibration of the thermoneedle in test measurements to achieve better accuracy and to offset the disadvantages of this technique explained in Sec. IV B 4. 


\section{Morphology}

\section{Morphology change trigger and observation system}

As shown in Table III, the chamber is equipped with five ISO-F 100 flanges, which allow observation of the samples at angles of $45^{\circ}$ and $64^{\circ}$. These flanges are equipped with viewing ports, which allow illumination and observation from different viewing angles. For the illumination, a second smaller solar simulator or a high-power lamp with up to ten solar constants will be used. Both devices can be mounted at all five windows and can be moved in a very short time even if the experiment is running. Also the main solar simulator can be used for illumination for the cameras. This setup gives the option to illuminate a specific spot on the sample, for example, a prepared cliff or a hole in the sample. In addition, this setup allows us to simulate different times of the cometary day by selective illumination of the sample.

For the observation of sample changes, a monochromatic camera, which can be mounted at any viewport, with a resolution of $3072 \times 2048$ pixel $^{2}$, is used. In standard experiments, this camera will observe the whole sample surface and monitors surface changes that may occur during an experiment run. For experimental campaigns dedicated to measuring the evolution of surface structures, the camera can be focused on a specific surface spot by changing the lens. As an example, a cliff with $50 \mathrm{~mm}$ height might be prepared in the middle of the sample. This cliff can then be illuminated, and it can be observed whether overhangs develop due to the outgassing of the sample.

\section{Mobile stereo imaging system}

In addition to the monochromatic camera mounted outside the chamber, two further cameras are mounted inside the cooling shield. They are attached to the Z-stage of the penetrometer and are referred to as the mobile stereo imaging system (see Sec. IV A 2). The size of these cameras is $29 \times 29 \times 29 \mathrm{~mm}^{3}$ and they weigh $75 \mathrm{~g}$, which makes them ideal for the limited space inside the cooling shield. They are linked to the Z-stage by aluminum arms, which can be adjusted to any position before the experiment is started. During the experiments, the cameras can be moved to investigate all surface areas of the sample. The cameras have a resolution of $4608 \times 3288 \mathrm{pixel}^{2}$, are color resolved, and can take up to 7 frames/s. For illumination, a ring holding multiple light emitting diodes is mounted around the lens.

The main advantage of the cameras inside the chamber is that a high spatial resolution can be achieved and that no reflection from the windows is present. Since the aluminum arms, holding the cameras, are fully adjustable, it is also possible to observe a specific item, like a cliff from two different viewing axes.

The cameras inside the vacuum chamber are not actively cooled, but their operation generates heat. This means that the cameras can be only used to monitor the samples for a short time period. This is why the monochromatic camera outside the chamber can be used to complement these measurements. The temperature of the cameras will be carefully monitored, and they are automatically switched off before overheating. The cameras are surrounded by aluminum foil to avoid heating of the samples. The heat from the cameras is transferred into the steel rail of the Z-stage, which has enough heat capacity to not significantly heat up by the camera operation. To avoid damage by the cold temperatures, the cameras are turned on and off periodically.

\section{Gas and dust emission \\ 1. High-speed tracking cameras}

The 3D-PTV technique offers the option to identify particles by their shadow or by the scattered light. Shadowgraphy is advantageous for identifying complex particle structures; however, it is, in general, only possible if the particles are at least the size of the pixel resolution, which means in our case between 10 and $50 \mu \mathrm{m}$, depending on the lens. In addition, this technique requires opposition of the camera and light source. Since the two main viewports for the high-speed cameras are placed at a $90^{\circ}$ angle to each other and opposite to those of the TMP and the door of the cooling shield, the shadowgraphy technique cannot be applied without adapting the L-Chamber.

In contrast, scattered light can be used at an arbitrary orientation from the cameras. The intensity is usually higher when the cameras record light in the near-forward-scattering direction. For either approach, particle detection rates are improved when the illumination duration and intensity is optimized for the recording frame rate of the camera. This optimization involves checking the pixel displacement between subsequent frames and setting a criterion for the maximum amount of blurring of the particles in the image. In order to obtain sub-pixel accuracy in the 2D-image plane, short-duration, monochromatic light is appropriate. CoPhyLab will use a light intensity of $\sim 7 \mathrm{~mJ} /$ pulse at a frequency of $1 \mathrm{kHz}$. The selected light source is an ILA 5150s LPS 3. In a typical experiment run with the standard geometry, this is equivalent to a total energy input of $14 \mathrm{~J} / \mathrm{h}$; hence, the sample is not disturbed by the illumination. This light source can be installed at any free viewport, which means that several different illumination configurations are possible. The standard configuration is shown in Fig. 16. The cameras are perpendicular to each other and the light source is positioned at an horizontal angle of $135^{\circ}$ and a vertical angle of $72^{\circ}$ to both cameras. The tracked particles are inside a volume, which is defined by the intersection of both fields of view from the cameras and the illumination plane of the light source. The selecting specific parts of the camera pictures the particles can be confined to a defined part of the sample surface, which is not illuminated be the light source; hence, the activity is not caused by the tracking setup itself. Since the experiments last for several days or weeks, monitoring the activity requires an automated recording mechanism. In our case, the system will be triggered if other instruments, for example, the scale or the pressure sensors, detect hints of activity. The camera system will measure with a frame rate up to $4 \mathrm{KHz}$ and at a resolution of $1000 \times 1000$ pixel $^{2}$. Because of the different windows installed on the chamber, the illumination conditions can be changed during the runs. The illumination itself will be synchronized with the cameras to allow for instant illumination when the pictures are recorded. Based on these requirements, we chose two Photron Fastcam AX100 Mini cameras, each with a 200 $\mathrm{mm}$ Nikkor Macro objective, timing unit, Q-switched light source, and portable computer server.

In order to make the system usable for the 3D-PTV technique, it is carefully calibrated by using a target mask in the position of the sample. Due to the required high precision, it is necessary to mount the cameras on reinforced plates so that no bending will occur due to the weight of the cameras. After the recording of the twodimensional images, the following steps are required to construct the three-dimensional trajectories: 


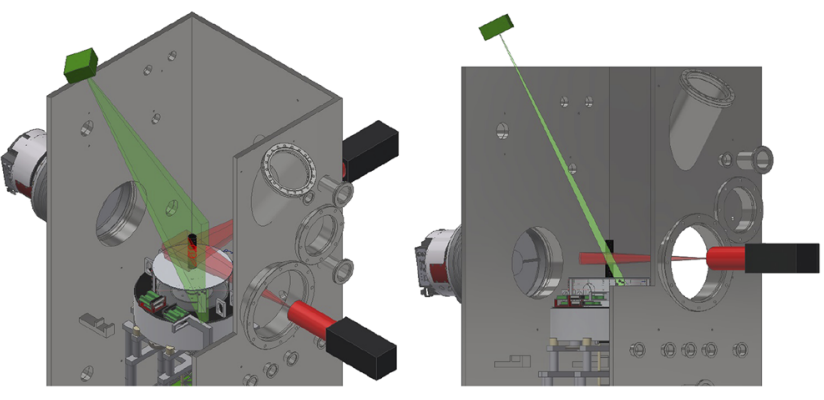

FIG. 16. Sketch of the 3D-PTV setup with two high-speed cameras (black box with red lens) and the light source (green box). The fields of view from both cameras are shown in transparent red, and the illumination plane of the light source is shown in transparent green. The cameras are installed in front of the two main viewing ports at the same height and at an angle of $90^{\circ}$ to each other. The light source is mounted at a horizontal angle of $135^{\circ}$ and a vertical angle of $72^{\circ}$ to both cameras. The black post in the center of the sample is showing a volume with $3 \mathrm{~cm}$ diameter in which the particles are flying upward from the sample surface. Only particles inside of both fields of view and the illumination plane can be tracked. By selecting specific parts of the camera images, the volume in which particles are tracked can be restricted to a volume above a defined part of the surface, in this case inside the black post. In the left picture, the geometry is shown from a $45^{\circ}$ angle, and the volume in which the particles are tracked is highlighted in red. The right picture shows the geometry from the side. Here, it is shown that the light source is not illuminating the area from which the particles are coming and that the particles are tracked in a defined height above the sample surface. In both pictures, the cooling shield is removed and the chamber wall is cut out for a clear view.

1. image acquisition and pre-processing (e.g., sliding background subtraction, ${ }^{60}$ image inversion, filtering, etc.);

2. refined particle self-calibration to minimize discrepancy vectors (difference between calculated position and highest correlation for hypothetical 3D-displacement vector);

3. shake-the-box algorithm ${ }^{61}$ to construct particle trajectories; and

4. write out $3 \mathrm{D}$ vectors of position, velocity, and acceleration for every particle at every time.

\section{Scale}

The scale used in the L-Chamber is a $P B K 989-A B 15$ model from Mettler Toledo. It has a maximum capacity of $15 \mathrm{~kg}$, a resolution of $0.02 \mathrm{~g}$ over the full range, and a time resolution of $12 \mathrm{~Hz}$. It is not specifically designed for use in vacuum, and therefore, it had to be tested in vacuum for extended periods of time. The scale shows significant fluctuations, around $2 \mathrm{~g}$, during the evacuation process due to the air flow. Once a stable pressure is reached, the scale can be operated normally. We measured the drift of the scale in vacuum by placing a $12 \mathrm{~kg}$ test weight onto the scale. The observed drift is $1.22 \mathrm{~g}$ in 3 weeks. The scale is thermally isolated from the sample holder by a TECAPEEK construction with a very low thermal conductivity. The internal electronics keep the scale warm enough to prevent damage and to measure accurately.

The scale rests on a scale lifting system installed at the bottom plate of the L-Chamber. It consists of slides (see red objects in Fig. 17) made from a special low-friction sliding material provided by Igus. They are pushed by two Nema23 stepper motors from opposite directions under a stainless-steel plate to lift the whole system
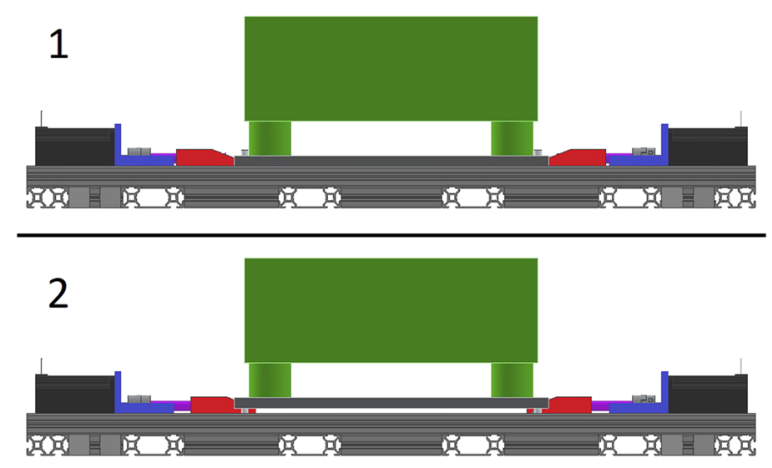

FIG. 17. Sketch of the bottom plate with the scale lifting system. The scale rests on a stainless-steel plate, which is lifted by pushing two slides (red) under it. In the top picture (a), the scale is lowered and detached from the sample holder. In the bottom picture (b), the scale is lifted by $5 \mathrm{~mm}$ and in the operating position.

upward. In the lifted position, the sample holder is detached from the sample cooling system and the scale can measure the weight of the sample. In this state, the sample holder is entirely cooled by radiation. In the lower position of the scale lifting system, the copper plate of the sample holder rests directly on the donut cooling system, which means that the scale cannot measure the weight of the sample, but the penetrometer can now be used without damaging the scale. The direct mechanical contact can be also used to increase the cooling rate of the sample holder.

The scale will be used to monitor the total mass loss during the full experiment run. Combined with the data from the mass spectrometer (see Sec. IV D 3), which measures the gas emission, it is possible to determine the dust mass loss. Furthermore, by using pure ice samples, both instruments can be cross-calibrated. The high temporal resolution of the scale provides the possibility to measure the mass loss during ejection events, which can be also used as a signal to trigger the high-speed cameras. In the case of the pure ice samples, the scale can be also used to measure the surface temperature by relating the ice mass loss rate to the Hertz-Knudsen equation. This method will be used to calibrate the mass spectrometer.

\section{Mass spectrometer}

To measure the outgassing rate, we decided to use a combined system that consists of a mass spectrometer (PrismaPlus QME220 from Pfeiffer Vacuum Components and Solutions $\mathrm{GmbH}$ ) with a mass range from 1 to $100 \mathrm{u}$ and a rotating chopper similar to the technique discussed by Gundlach, Skorov, and Blum. ${ }^{62}$ Whenever the rotating chopper opens, the mass spectrometer can directly observe the sample and the signal measured is proportional to the sum of the background and the momentum flux from the sublimating molecules. In the closed state, the mass spectrometer only measures the background pressure. Hence, subtraction and integration of both signals, which is typically performed by a lock-in amplifier, provides a signal that is directly proportional to the outgassing rate. Because we favor the use of a mass spectrometer over a pressure sensor, this setup provides also the outgassing rates of different volatiles simultaneously. The calibration with the known surface temperature of the ices is required to relate the measured currents to the real outgassing rates. Once calibrated, the system can be operated 
independently and can measure all sample types as long as the pressure inside the chamber is below $8 \times 10^{-5} \mathrm{mbar}$, and therefore, the mean free path of the molecules is larger than $1150 \mathrm{~mm}$, which is the distance between the sample surface and the mass spectrometer. A similar system is currently used in a smaller chamber, and the results are currently written up in a separate paper.

\section{E. Optical properties}

During the sublimation experiments, the properties of the sample's surface will be monitored using either of the two imaging systems. Moreover, an optical fiber setup will be employed to follow the spectral changes in the sample surface or as support for a secondary illumination system.

\section{Mobile hyperspectral imaging setup}

The mobile hyperspectral imaging setup (MoHIS), attached to flange B8 (see Table III) and looking from the right hand side on to the sample (right green line in Fig. 18), will be used to acquire hyperspectral visible-near infrared (VIS-NIR) data cubes with a spectral resolution better than $10 \mathrm{~nm}$. This system can only be operated when the solar simulator is turned off as it uses its own monochromatic light source.

The instrument is based on the system as first set up on the Bernese SCITEAS simulation chamber ${ }^{63}$ and has been extensively modified to make it portable and adaptable to different chamber configurations while its performance was regularly improved. ${ }^{64}$ It consists of a $250 \mathrm{~W}$ halogen lamp and a grating monochromator coupled to a fiber bundle with $5 \mathrm{~mm}$ diameter to illuminate the samples with narrow bandpasses of light. Furthermore, a visible camera with a $1392 \times 1040$ pixel $^{2}$ CCD detector and an average image scale of $0.46 \mathrm{mrad} \times$ pixel $^{-1}$ is included. In addition, MoHIS has an infrared camera with a $320 \times 256$ pixel $^{2}$ MCT detector and an average image scale of $2.25 \mathrm{mrad} \times$ pixel $^{-1}$.

Using either the CCD or the MCT camera, the system can map the surface of the sample at wavelengths ranging from 380 to 1055 $\mathrm{nm}$ and from 800 to $2450 \mathrm{~nm}$ at spectral resolutions as low as $6 \mathrm{~nm}$ and variable wavelength steps. The default selection of wavelengths has been chosen in order to target the absorption maxima of the ice and the dust components while balancing spectral and temporal resolutions in previous sublimation experiments. ${ }^{65}$ However, the corresponding monochromator configuration can be easily modified, and the sampling of these wavelength ranges can be adapted to match the appropriate temporal resolution for a particular sublimation experiment.

The analysis of the hyperspectral cubes will allow us to follow and map the evolution of the ice-to-dust ratio across the sample's surface by analyzing the visible albedo as well as the steepness of the spectral slope in the visible electromagnetic spectrum and fitting of the strong and distinctive absorption bands of water ice in the NIR part of the spectrum. Examples of datasets and their use and interpretations can be found in the work of Pommerol et al., ${ }^{63}$ Poch et al., ${ }^{66,67}$ Jost et al., ${ }^{68}$ and Yoldi et al. ${ }^{69}$ For experiments using both water ice and carbon dioxide ice, these measurements will be crucial to identify the type of ices potentially exposed at the surface.

\section{Mobile and modular visible imaging setup}

The mobile and modular visible imaging setup (MoMoVIS) will be used to perform broadband photometry in the visible spectrum

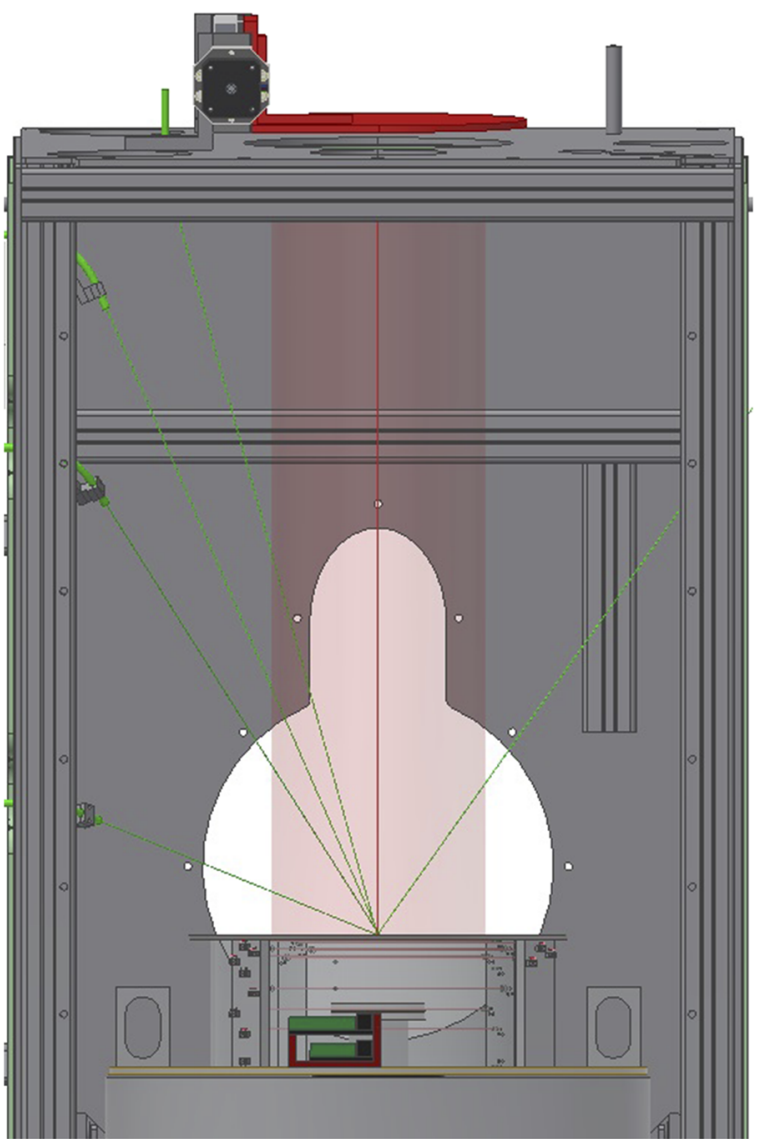

FIG. 18. Schematic drawing of the viewing axes of the optical instruments inside the chamber (the penetrometer is not shown for better visibility). In red, the light beam of the solar simulator is shown with the thin solid line marking the center of the light beam. From the right side, the MoHIS and MoMoVIS instruments are viewing the sample at an angle of $36^{\circ}$. From the left side, the four optical fibers illuminate or observe the sample surface at angles of $15^{\circ}, 27^{\circ}, 44^{\circ}$, and $75^{\circ}$ relative to the solar simulator beam. All viewing axes intersect in the plane of the sample surface.

with a high spatial resolution. While the spectral-imager MoHIS can only operate during night cycles, MoMoVIS will be used when the solar simulator is turned on or when fiber-coupled LEDs (see Sec. IV E 3) are used. This instrument, developed specifically for this project, is composed of a monochromatic camera with a mounted F2.8/50 mm lens and a six-slot filter wheel. The instrument will also be placed in front of flange window $\mathrm{B} 8$.

The camera has a $2048 \times 2048$ pixel $^{2}$ CCD detector sensible in a wavelength window ranging from 350 to $900 \mathrm{~nm}$, while the filter wheel is equipped with six high-transmission bandpass filters. The central wavelength and the full-width at half-maximum are given in Table IV. The choice of the bandpass filters centered at these particular wavelengths is the best compromise between the manufacturer's offers, the specifications of the Bessell filters, ${ }^{70}$ which are typically used in broadband photometry, and the specifications of the filters used for the ESA/OSIRIS-NAC instrument. A near-UV filter 
TABLE IV. Central wavelengths and full-widths at half-maximum of the selected filters for MoMoVIS.

\begin{tabular}{lcc}
\hline \hline $\begin{array}{l}\text { Filter } \\
\text { number }\end{array}$ & $\begin{array}{c}\text { Central } \\
\text { wavelength }(\mathrm{nm})\end{array}$ & $\begin{array}{c}\text { Full-width at } \\
\text { half-maximum }(\mathrm{nm})\end{array}$ \\
\hline 1 & 355 & 10 \\
2 & 400 & 40 \\
3 & 520 & 40 \\
4 & 650 & 40 \\
5 & 750 & 40 \\
6 & 850 & 40 \\
\hline \hline
\end{tabular}

was chosen to investigate the formation of water frost deposits, as observed on the comet 67P/Churyumov-Gerasimenko. ${ }^{71-73}$

Cross-calibrated with MoHIS and using the same analysis methods as for MoHIS's hyperspectral cubes, MoMoVIS will be used to estimate, map, and monitor the water-ice content across the surface based on the inversion of the spectro-photometric properties of the sample in the visible spectrum. This will be possible because the mixture's properties will have been fully characterized following the methods described in Ref. 53 and the work of Feller et al. ${ }^{7}$

\section{Optical-fibers}

In addition to MoHIS and MoMoVIS, the L-Chamber will be equipped with four optical fibers. The fibers are installed through flange D6 and will be used either to provide an illumination system or to allow spectroscopic measurements. Four multi-mode stepindex optical fibers with a numerical aperture of 0.22 , i.e., a solid angle of $1.54 \times 10^{-1} \mathrm{sr}$, are fixed on the left and top plate of the cooling shield, pointing toward the center of the sample. Considering the dimensions of the L-Chamber and the fibers' numerical aperture, the cone of vision of these optical fibers will intercept the entire surface of the sample. When using these optical fibers, the sides of the sample holder will be covered with black masking tape to avoid spurious light reflections.

While the angle between the centers of the E7 and B8 flanges is about $36^{\circ}$, the angles of the four optical fibers with respect to the flange $\mathrm{B} 8$ are about $51^{\circ}, 63^{\circ}, 75^{\circ}$, and $102^{\circ}$. In Fig. 18 , the viewing axes of the four optical fibers, the MoHIS and MoMoVIS instruments, and the solar simulator are shown. Theses angles where chosen to allow a comprehensive modeling of the spectro-photometric properties while avoiding the penetrometer inside the cooling shield.

As indicated previously, the optical fibers will be used either as part of a spectral monitoring setup or as an alternative secondary illumination system. The latter shall be configured depending on the experiment and the instruments conjointly used. Either a system of fiber-coupled LEDs (emitting at wavelengths matching the MoMoVIS bandpass filters) will be attached to the fibers or, instead, a white-laser or the monochromator of MoHIS could be used to provide an illumination at any given wavelength in the VNIR domain.

Alternatively, two different commercial slit-spectrometers from OceanOptics can be connected to the fibers. The first option is a USB2000 covering the wavelength range from 250 to $880 \mathrm{~nm}$, and the second option is a NIR512 covering the $850-1700 \mathrm{~nm}$ wavelength range. The USB2000 has a $1 \times 2048$ pixel $^{2}$ CCD detector with a spectral resolution of $0.4 \mathrm{~nm} /$ pixel, and the NIR512 has a $1 \times 512 \mathrm{pixel}^{2}$
InGaAs detector with a spectral resolution of $3.8 \mathrm{~nm} /$ pixel. These spectrometers will allow us to measure the sample's reflectance spectrum across the illuminated surface. In that case, the phase angles between the optical fibers and the solar simulator would be about $15^{\circ}, 27^{\circ}, 44^{\circ}$, and $75^{\circ}$, respectively. Hence, these spectrometers will allow us to measure continuously the global surface composition of the illuminated area while following the progressive disappearance of the $1.5 \mu \mathrm{m}$ water-ice absorption band during the sublimation process. Any such spectral changes would be compared to the findings of the MoHIS or MoMoVIS instruments to form a comprehensive picture of the sample's morphology, its spectro-photometric properties, and the compositional and physical properties of its surface layers.

\section{SAMPLE PREPARATION AND TRANSPORT}

\section{A. Sample preparation}

The samples for all experiments to be conducted in the LChamber will be produced directly within the sample holder either on the copper plate or in the steel sample holders for the Brazilian disk test. Therefore, a special glove-box, shown in Fig. 19, was designed for the sample preparation. The size of the glove-box is $1200 \times 700 \times 800 \mathrm{~mm}^{3}$ and it has a total of four removable plates. Two plates are used to move the sample holder and sample material

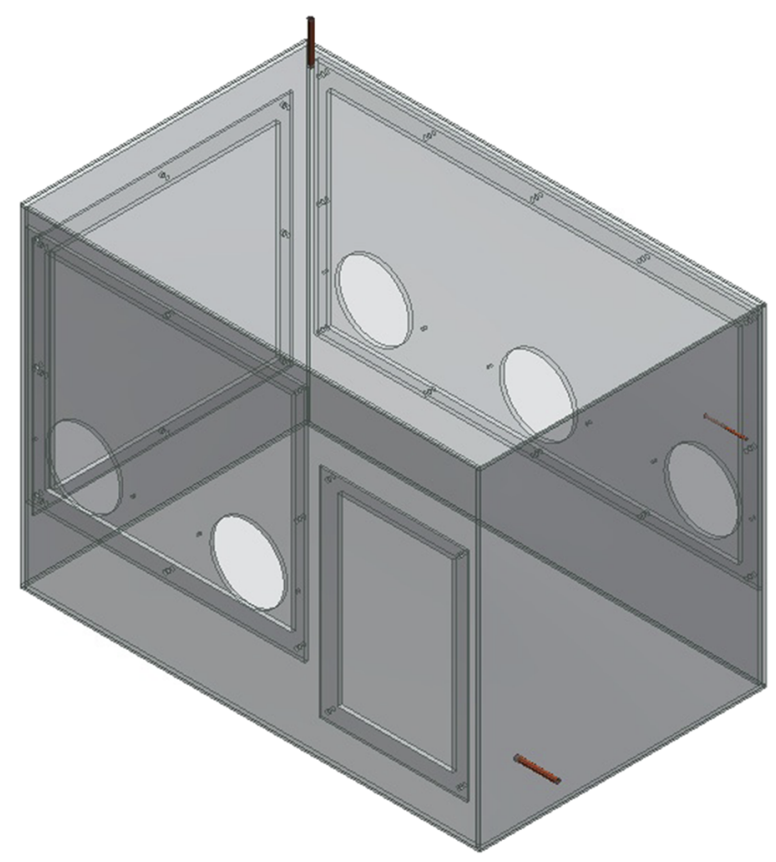

FIG. 19. Sketch of the glove-box viewed from the front under a $45^{\circ}$ angle. The glove-box is made of Plexiglas with a thickness of $5-10 \mathrm{~mm}$. A total of four doors can be removed for sample transfer and for adjusting the box to different sample sizes. Therefore, the two plates including the openings for the gloves can be turned by $180^{\circ}$ so that the height of the openings can be changed by $300 \mathrm{~mm}$. Furthermore, the glove-box has gas inlets and outlets to always be flushed with nitrogen gas. A third inlet is used to transport liquid nitrogen for sample cooling during sample preparation. 
into the glove-box. The other two plates on opposite sides contain the openings for the gloves. Two openings are installed on the front side and three on the rear side. The design allows for two persons to work simultaneously on the sample preparation. In addition, the person working on the rear side can use the extra opening for easy access to the sample materials. The openings are located in removable plates that can be turned by $180^{\circ}$ so that the height of the openings can be changed by $200 \mathrm{~mm}$. This is important because the sample height can vary between $50 \mathrm{~mm}$ for the Brazilian disk test and $300 \mathrm{~mm}$ for the tallest sublimation samples. Therefore, flexibility is crucial to allow the preparation of all planned sample sizes.

Inside the glove-box, six separate boxes are placed, which are filled with liquid nitrogen. One of them contains the sample holder and the others the sample materials and the tools needed for preparation. The liquid nitrogen cools the sample holder from below so that the sample is never in direct contact with liquid nitrogen. The sample materials, for example, water ice, carbon dioxide ice, silica dust, or charcoal, will be stored in CF tubes (vacuum tubes and blind flanges sealed with a copper seal) made from stainless steel. The sample material production will be described in a future paper in detail. The CF tubes protect the sample materials and they are very easy to handle, even with gloves. Also the high thermal inertia of the stainless steel tubes ensures low temperatures even if the tube is momentarily not actively cooled. All ingredients and all parts in contact with these are constantly kept below $110 \mathrm{~K}$. The liquid nitrogen required for the sample preparation is directly pumped into the glove-box. Nitrogen gas is constantly pumped into the glove-box to avoid the formation of frost.

All PT1000 sensors as well as the heat stripe and potential other sensors, which are located inside the sample, are placed in the sample holder before the sample is produced. This is especially important because the location of all physical sensors inside the sample must be known precisely to avoid damaging them with the penetrometer. Furthermore, the exact location of all PT1000 sensors is important for the thermophysical models. All sensors are checked before the samples are produced, since the extreme conditions can cause damage over time. The sample materials are then placed inside the sample holder. This can be done in layers or homogeneously mixed, depending on the experiments planned. In the end, the surface of the sample can be textured so that, e.g., cliffs can be created. After that, the sample is covered to be protected during transport.

\section{B. Sample transport}

For the transport of the sample from the glove-box to the LChamber, a special transport cart was developed. This was necessary because the weight of the sample holder with the sample inside is about $13 \mathrm{~kg}$ and high precision is needed to place the sample holder in its foreseen position inside the sample cooling system (see Sec. III D) without touching it. Furthermore, the sample must be kept below $110 \mathrm{~K}$ to avoid desiccation and sintering effects and the mechanical stresses applied to it must be minimized in order to preserve the structure of the material.

The chosen design for the transport cart, shown in Fig. 20, is adapted from a fork lifter. It is made out of three main aluminum frames and a moving arm, which can be pushed out by $250 \mathrm{~mm}$. The bottom frame holds a stepper-motor-driven rail and has wheels attached to it. The upper two frames are bolted together and in

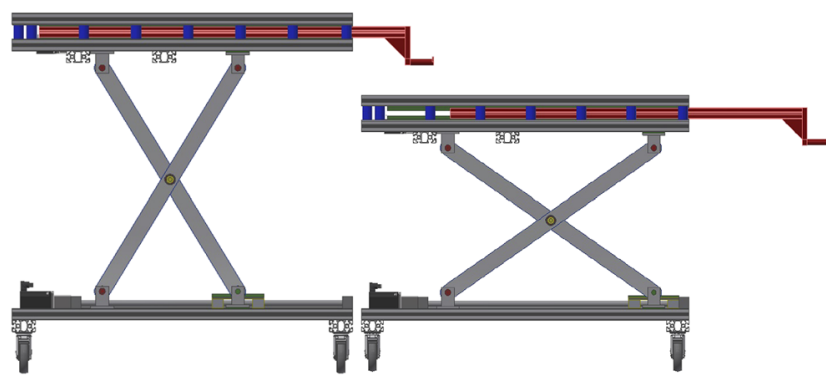

FIG. 20. Sample transport cart in two different positions. On the left, the lower stepper-motor-driven rail was used to lift the upper frame. The moving arm is pulled in using the upper stepper-motor-driven rail. This position is used to pick the sample up inside the glove-box. On the right, the upper frame is lowered and the moving arm is extended. In this position, the sample is placed on the sample cooling system.

between them, and the moving arm is mounted. A second steppermotor-driven rail is mounted to this frame unit. This rail is connected to the moving arm and can be positioned by a Nema23 stepper motor. Between the two frame units, four aluminum posts are mounted, which form two crosses on both sides of the frames. On the rear side, the posts are fixed to the frame, and on the front side, they can slide along rails in the frames. On the lower frame, the two loose ends are connected to the slider of the stepper-motor-driven rail powered by a Nema34 motor and can be pulled along with it. By moving the loose ends closer to the fixed ends, the upper frame unit is lifted upward.

For the transport of the sample, the sample holder is picked up by two metal bars, located on the moving arm, and can be lifted out of the glove-box. After that, the cart is driven slowly toward the chamber to avoid vibrations. Liquid nitrogen is stored in a second containment around the sample on the sample holder to actively cool the sample during transport. This evaporating liquid nitrogen also creates a dry nitrogen-gas atmosphere, which protects the sample from frost condensation. After the cart is parked in a fixed position, it is programmed to place the sample directly in the cooling system on the holding structure below. Once the sample holder is placed inside the L-Chamber, the transport cart is removed, all cables are connected to the sample holder, the sample cover is removed, and the cooling shield door and the main vacuum chamber door are closed.

\section{L-CHAMBER PREPARATION AND OPERATION}

\section{A. L-Chamber preparation}

Before an active experiment, a set of preparatory steps is required to condition the L-Chamber. The first step is to evacuate the L-Chamber and to pre-cool the cooling shield and the sample cooling system. Due to the high masses of these systems, this task must be done well before the sample is placed inside the chamber; otherwise, the temperature of the sample would rise above the sintering temperature of the ices and the sample properties would change. After the sample is prepared and both cooling systems have reached a steady state temperature level, the chamber is flooded with argon gas to avoid the formation of frost on the cold metal parts. Frost 
would be particularly harmful to the sample cooling system, since it reduces the efficiency of the radiative cooling. Furthermore, frost could also condense inside the small gap between the cooling system and the sample holder, which would affect the scale measurements. After ambient pressure is reached inside the chamber, the door is opened and the sample is placed inside the chamber as described in Sec. V. Since the sample cooling system is located at a lower position than the door, the heavy argon gas protects it even after the door is opened. In order to protect the cooling shield in the upper half of the chamber, dry synthetic air is blown into the chamber through flange E5 (see Sec. III). This ensures an over-pressure with dry air inside the chamber, which warrants that only minimal moisture can enter the L-Chamber.

\section{B. L-Chamber operation}

Once the L-Chamber is closed, the evacuation begins by opening the electronically controlled valve. The aim is to pump as fast as possible without destroying the highly porous samples. The sample evolution during the evacuation phase is also monitored by cameras so that the pumping speed can be adjusted if the samples tend to be altered. The measurements are initiated when the temperatures and pressure reached a steady state.

The duration of the experimental runs in terms of chamber operation is, in principle, unlimited, as long as the cooling systems are supplied with liquid nitrogen, but still samples might be only interesting for a limited time span due to sample alteration. The liquid-nitrogen cans are placed on scales, which are connected to the control computer. This allows us to measure the amount of liquid nitrogen still present in the cans. All collected data are automatically captured by the main computer, and only certain instruments, such as the penetrometer and the heatstrip, must be operated manually. These measurements will have to follow a predetermined protocol, since they can interfere with each other. This is especially important for the penetrometer, since it produces a shadow on the sample surface and the scale lifting device must be lowered before the penetrometer is used.

\section{L-Chamber performance test}

The L-Chamber was tested without a sample to determine the baseline for the pressure and temperatures that can be reached. For this test, the sample holder was placed on its TECAPEEK construction without having contact to the main sample cooling system and the test was started at room temperature. The full temperature plot is shown in Fig. 21. In the beginning, the chamber was evacuated to $8 \times 10^{-6}$ mbar and then the cooling shield was filled with liquid nitrogen. This was done to check if the cooling shield is leaking nitrogen into the chamber and thereby increasing the pressure, which is not the case. The temperature of the sample holder (red line) was dropping slowly in the time the cooling shield was filled up. Subsequently, the sample cooling system was started and its temperature (blue dashed line) dropped to liquid nitrogen temperature in a few minutes and stayed constantly at this level. The sample holder, which was only cooled by radiation, increased its cooling rate at this point and reached its minimal temperature of $128.1 \mathrm{~K}$ after $48 \mathrm{~h}$. The test was ended after two full days although the temperature was still dropping because the target temperature of $150 \mathrm{~K}$, which is sufficient for keeping water ice samples from sintering, ${ }^{1}$

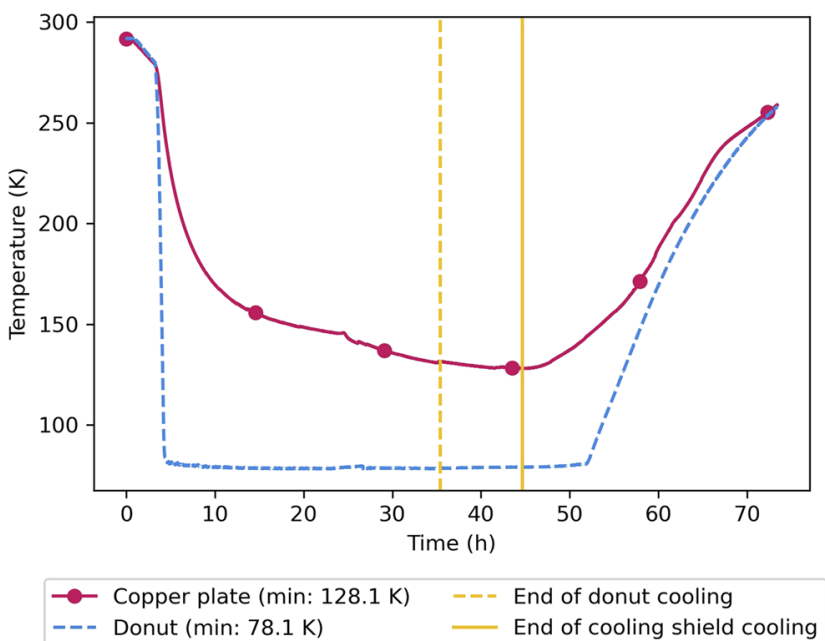

FIG. 21. Measured temperatures of the copper sample holder and the top surface of the sample cooling system. This cooling test was started with the sample holder at room temperature, and for hours, only the cooling shield was cooled, leading to a steady drop in temperature. The sample cooling system was started after two hours, and its temperature dropped to liquid nitrogen temperature very fast and stayed constant. The sample holder cooled down to $128.1 \mathrm{~K}$ in $48 \mathrm{~h}$. The dashed yellow line marks the time the sample cooling system was stopped, and the solid yellow line indicates the stop of the cooling shield. It takes $13 \mathrm{~h}$ for the sample cooling system to start warming up after the nitrogen supply is stopped. The pressure during the test was stabilized at $8 \times 10^{-6} \mathrm{mbar}$.

was reached. After both cooling systems reached a stable temperature, the nitrogen consumption was $7.2 \frac{\mathrm{kg}}{\mathrm{h}}$ for the sample cooling system and $10.3 \frac{\mathrm{kg}}{\mathrm{h}}$ for the cooling shield. The nitrogen for the cooling systems is supplied from two nitrogen cans holding $180 \mathrm{~kg}$ each. This means that long-term experiments can be conducted without having to fill up nitrogen in the middle of the night. The test also showed that the sample cooling systems takes $13 \mathrm{~h}$ to start warming up after the nitrogen supply is stopped, hence ensuring a stable state of the sample even if the cooling system is stopped temporally. During the whole test, the pressure was at $8 \times 10^{-6}$ mbar with little fluctuation after the sample cooling system was started. This came from a small leak, which is known and will be sealed in the future. Overall, the test successfully proved that the L-Chamber can keep a sample below $150 \mathrm{~K}$ and below the critical pressure for the mass spectrometer of $8 \times 10^{-5} \mathrm{mbar}$. The long cooling time in this test is not relevant since the sample will be placed in the chamber with a temperature of $100 \mathrm{~K}$, so the cooling systems only have to keep the sample cold.

\section{SUMMARY}

The CoPhyLab L-Chamber described in this work allows for the exploration of cometary physics at a great level of detail. The experiments that can be performed inside the L-Chamber aim to improve on previous cometary experiments. Weaknesses of previous studies have been considered when designing the L-Chamber. The temperatures and pressure reached by the system $\left(130 \mathrm{~K}\right.$ and $\left.10^{-6} \mathrm{mbar}\right)$ will 
allow us to study and better understand cometary physics in a realistic cometary environment. Cometary processes that are not fully understood even after Rosetta (Keller and Kührt ${ }^{74}$ ) can be studied under lab conditions. This will provide new insight into open scientific questions as the consequences of mechanical properties of the matter, the dust-to-ice ratio, and morphologic features for cometary activity and as the transport mechanisms for the lift-off of dust grains from the surface. The CoPhyLab L-Chamber instruments will allow the study of mechanical properties, the internal and surface temperatures of the sample, the changes of morphology due to illumination, the gas and particle ejection form the surface, as well as the changes of the optical properties of the surface. These measurements can then be compared to cometary missions in order to better understand the processes that take place on comets as they orbit the Sun. The L-Chamber is planned for short- and long-duration experiments in a safe and controlled environment, the data produced is planned to be made available to the public, and the chamber can be used in the future by the planetary science community to study many different samples under different conditions.

\section{ACKNOWLEDGMENTS}

This work was carried out in the framework of the CoPhyLab project funded by the D-A-CH program (Grant Nos. DFG GU 1620/3-1, BL 298/26-1, SNF 200021E 177964, and FWF I 3730-N36).

\section{DATA AVAILABILITY}

Data sharing is not applicable to this article as no new data were created or analyzed in this study.

\section{REFERENCES}

${ }^{1}$ P. Weissman, A. Morbidelli, B. Davidsson, and J. Blum, "Origin and evolution of cometary nuclei," Space Sci. Rev. 216, 6 (2020).

${ }^{2}$ A. Johansen, J. S. Oishi, M.-M. Mac Low, H. Klahr, T. Henning, and A. Youdin, "Rapid planetesimal formation in turbulent circumstellar disks," Nature 448, 1022-1025 (2007); arXiv:0708.3890 [astro-ph].

${ }^{3}$ Y. Skorov and J. Blum, "Dust release and tensile strength of the nonvolatile layer of cometary nuclei," Icarus 221, 1-11 (2012); arXiv:1111.0768 [astro-ph.EP].

${ }^{4}$ J. Blum, B. Gundlach, S. Mühle, and J. M. Trigo-Rodriguez, "Comets formed in solar-nebula instabilities!-An experimental and modeling attempt to relate the activity of comets to their formation process," Icarus 235, 156-169 (2014); arXiv:1403.2610 [astro-ph.EP].

${ }^{5}$ J. Blum, B. Gundlach, M. Krause, M. Fulle, A. Johansen, J. Agarwal, I. von Borstel, X. Shi, X. Hu, M. S. Bentley, F. Capaccioni, L. Colangeli, V. Della Corte, N. Fougere, S. F. Green, S. Ivanovski, T. Mannel, S. Merouane, A. Migliorini, A. Rotundi, R. Schmied, and C. Snodgrass, "Evidence for the formation of comet 67P/Churyumov-Gerasimenko through gravitational collapse of a bound clump of pebbles," Mon. Not. R. Astron. Soc. 469, S755-S773 (2017); arXiv:1710.07846 [astro-ph.EP].

${ }^{6}$ B. Davidsson, S. A. Stern, W. Kofman, M. Hilchenbach, A. Rotundi, M. Bentley, M. Hofstadter, H. Sierks, K. Altwegg, H. Nilsson, J. L. Burch, A. I. Eriksson, K.-H. Glassmeier, P. Henri, C. M. Carr, M. Pätzold, F. Capaccioni, H. Boehnhardt, J.-P. Bibring, M. Fulle, M. Fulchignoni, E. Gruen, P. R. Weissman, M. Taylor, B. J. Buratti, M. Choukroun, N. Altobelli, C. Snodgrass, and Rosetta Science Working Team, "The Rosetta mission: Clues on the origin of comet nuclei," in AAS/Division for Planetary Sciences Meeting Abstracts No. 48, AAS/Division for Planetary Sciences Meeting Abstracts (American Astronomical Society/Divison for Planetary Sciences, 2016), Vol. 48, p. 104.05.
${ }^{7}$ F. Windmark, T. Birnstiel, C. Güttler, J. Blum, C. P. Dullemond, and T. Henning, "Planetesimal formation by sweep-up: How the bouncing barrier can be beneficial to growth," Astron. Astrophys. 540, A73 (2012); arXiv:1201.4282 [astro-ph.EP].

${ }^{8}$ F. Windmark, T. Birnstiel, C. W. Ormel, and C. P. Dullemond, "Breaking through: The effects of a velocity distribution on barriers to dust growth (corrigendum)," Astron. Astrophys. 548, C1 (2012).

${ }^{9}$ O. V. Dobrovolskii and E. A. Kaimakov, "Surface phenomena in simulated cometary nuclei," in IAU Colloquium 39: Comets, Asteroids, Meteorites: Interrelations, Evolution and Origins, edited by A. H. Delsemme (International Astronomical Union, 1977), pp. 37-45.

${ }^{10}$ R. S. Saunders, F. P. Fanale, T. J. Parker, J. B. Stephens, and S. Sutton, "Properties of filamentary sublimation residues from dispersions of clay in ice," Icarus $\mathbf{6 6}$, 94-104 (1986).

${ }^{11}$ A. D. Storrs, F. P. Fanale, R. S. Saunders, and J. B. Stephens, "The formation of filamentary sublimate residues (FSR) from mineral grains," Icarus 76, 493-512 (1988).

${ }^{12}$ E. Grün, A. Bar-Nun, J. Benkhoff, A. Bischoff, H. Düren, H. Hellmann, P. Hesselbarth, P. Hsiung, H. U. Keller, J. Klinger, J. Knölker, H. Kochan, H. Kohl, G. Kölzer, D. Krankowsky, P. Lämmerzahl, K. Mauersberger, G. Neukum, A. K. Roessler, G. Schwehm, T. Spohn, D. Stöfler, and K. Thiel, "Laboratory simulation of cometary processes: Results from first KOSI experiments," in IAU Colloquium 116: Comets in the Post-Halley Era, edited by J. R. L. Newburn, M. Neugebauer, and J. Rahe (International Astronomical Union, 1991), Vol. 167, p. 277.

${ }^{13}$ E. Gruen, J. Benkhoff, and J. Gebhard, "Past, present and future KOSI comet simulation experiments," Ann. Geophys. 10, 190-197 (1992).

${ }^{14}$ G. Kölzer, E. Grün, H. Kochan, P. Lämmerzahl, and K. Thiel, "Dust particle emission dynamics from insolated ice/dust mixtures: Results from the KOSI 5 experiment," Planet. Space Sci. 43, 391-407 (1995).

${ }^{15}$ M. Choukroun, K. Altwegg, E. Kührt, N. Biver, D. Bockelée-Morvan, J. Drążkowska, A. Hérique, M. Hilchenbach, R. Marschall, M. Pätzold, M. G. G. T. Taylor, and N. Thomas, "Dust-to-gas and refractory-to-ice mass ratios of comet 67P/Churyumov-Gerasimenko from Rosetta observations," Space Sci. Rev. 216, 44 (2020).

${ }^{16}$ M. Fulle, N. Altobelli, B. Buratti, M. Choukroun, M. Fulchignoni, E. Grün, M. G. G. T. Taylor, and P. Weissman, "Unexpected and significant findings in comet 67P/Churyumov-Gerasimenko: An interdisciplinary view," Mon. Not. R. Astron. Soc. 462, S2-S8 (2016).

${ }^{17}$ M. Fulle, “The ice content of Kuiper belt objects," Nat. Astron. 1, 0018 (2017).

${ }^{18} \mathrm{H}$. U. Keller and E. Kührt, "On the formation of cometary surface crusts," in AAS/Division for Planetary Sciences Meeting Abstracts No. 25, AAS/Division for Planetary Sciences Meeting Abstracts (American Astronomical Society/Divison for Planetary Sciences, 1993), Vol. 25, p. 14.04.

${ }^{19}$ B. Gundlach, J. Ratte, J. Blum, J. Oesert, and S. N. Gorb, "Sintering and sublimation of micrometre-sized water-ice particles: The formation of surface crusts on icy solar system bodies," Mon. Not. R. Astron. Soc. 479, 5272-5287 (2018).

${ }^{20}$ B. Gundlach, K. P. Schmidt, C. Kreuzig, D. Bischoff, F. Rezaei, S. Kothe, J. Blum, B. Grzesik, and E. Stoll, "The tensile strength of ice and dust aggregates and its dependence on particle properties," Mon. Not. R. Astron. Soc. 479, 1273-1277 (2018).

${ }^{21}$ D. Haack, K. Otto, B. Gundlach, C. Kreuzig, D. Bischoff, E. Kührt, and J. Blum, "The tensile strength of dust-ice mixtures and their relevance as cometary analog material," Astron. Astrophys. 642, A218 (2020).

${ }^{22}$ D. Bischoff, C. Kreuzig, D. Haack, B. Gundlach, and J. Blum, "Sticky or not sticky? Measurements of the tensile strength of microgranular organic materials," Mon. Not. R. Astron. Soc. 497, 2517-2528 (2020); arXiv:2007.07538 [astroph.EP].

${ }^{23}$ I. L. San Sebastián, A. Dolff, J. Blum, M. G. Parisi, and S. Kothe, "The tensile strength of compressed dust samples and the catastrophic disruption threshold of pre-planetary matter," Mon. Not. R. Astron. Soc. 497, 2418-2424 (2020); arXiv:2007.08421 [astro-ph.EP].

${ }^{24}$ C. P. Opeil, G. J. Consolmagno, and D. T. Britt, "The thermal conductivity of meteorites: New measurements and analysis," Icarus 208, 449-454 (2010).

${ }^{25} \mathrm{~B}$. Gundlach and J. Blum, "Outgassing of icy bodies in the solar system-II: Heat transport in dry, porous surface dust layers," Icarus 219, 618-629 (2012). 
${ }^{26}$ X. Hu, X. Shi, H. Sierks, J. Blum, J. Oberst, M. Fulle, E. Kührt, C. Güttler, B. Gundlach, H. U. Keller, S. Mottola, M. Pajola, C. Barbieri, P. L. Lamy, R. Rodrigo, D. Koschny, H. Rickman, J. Agarwal, M. F. A'Hearn, M. A. Barucci, J.-L. Bertaux, I. Bertini, S. Boudreault, I. Büttner, G. Cremonese, V. Da Deppo, B. Davidsson, S. Debei, M. De Cecco, J. Deller, S. Fornasier, O. Groussin, P. J. Gutiérrez, P. Gutiérrez-Marqués, I. Hall, M. Hofmann, S. F. Hviid, W.-H. Ip, L. Jorda, J. Knollenberg, G. Kovacs, J. R. Kramm, M. Küppers, L. M. Lara, M. Lazzarin, J. J. López-Moreno, F. Marzari, G. Naletto, N. Oklay, M. L. Richards, J. Ripken, N. Thomas, C. Tubiana, and J.-B. Vincent, "Thermal modelling of water activity on comet $67 \mathrm{P} / \mathrm{Churyumov}-$ Gerasimenko with global dust mantle and plural dust-to-ice ratio," Mon. Not. R. Astron. Soc. 469, S295-S311 (2017).

${ }^{27}$ A. T. Basilevsky and H. U. Keller, "Comet nuclei: Morphology and implied processes of surface modification," Planet. Space Sci. 54, 808-829 (2006).

${ }^{28}$ A. F. Cheng, C. M. Lisse, and M. A'Hearn, "Surface geomorphology of Jupiter family comets: A geologic process perspective," Icarus 222, 808-817 (2013).

${ }^{29}$ N. Thomas, H. Sierks, C. Barbieri, P. L. Lamy, R. Rodrigo, H. Rickman, D. Koschny, H. U. Keller, J. Agarwal, M. F. A'Hearn, F. Angrilli, A.-T. Auger, M. A. Barucci, J.-L. Bertaux, I. Bertini, S. Besse, D. Bodewits, G. Cremonese, V. Da Deppo, B. Davidsson, M. De Cecco, S. Debei, M. R. El-Maarry, F. Ferri, S. Fornasier, M. Fulle, L. Giacomini, O. Groussin, P. J. Gutierrez, C. Güttler, S. F. Hviid, W.-H. Ip, L. Jorda, J. Knollenberg, J. R. Kramm, E. Kührt, M. Küppers, F. La Forgia, L. M. Lara, M. Lazzarin, J. J. L. Moreno, S. Magrin, S. Marchi, F. Marzari, M. Massironi, H. Michalik, R. Moissl, S. Mottola, G. Naletto, N. Oklay, M. Pajola, A. Pommerol, F. Preusker, L. Sabau, F. Scholten, C. Snodgrass, C. Tubiana, J.-B. Vincent, and K.-P. Wenzel, "The morphological diversity of comet 67P/Churyumov-Gerasimenko," Science 347, aaa0440 (2015).

${ }^{30}$ J. M. Sunshine, N. Thomas, M. R. El-Maarry, and T. L. Farnham, "Evidence for geologic processes on comets," J. Geophys. Res.: Planets 121, 2194-2210, https://doi.org/10.1002/2016je005119 (2016).

${ }^{31}$ M. R. El-Maarry, O. Groussin, H. U. Keller, N. Thomas, J.-B. Vincent, S. Mottola, M. Pajola, K. Otto, C. Herny, and S. Krasilnikov, "Surface morphology of comets and associated evolutionary processes: A review of rosetta's observations of 67P/Churyumov-Gerasimenko," Space Sci. Rev. 215, 36 (2019).

${ }^{32}$ A.-T. Auger, O. Groussin, L. Jorda, M. R. El-Maarry, S. Bouley, A. Séjourné, R. Gaskell, C. Capanna, B. Davidsson, S. Marchi, S. Höfner, P. L. Lamy, H. Sierks, C. Barbieri, R. Rodrigo, D. Koschny, H. Rickman, H. U. Keller, J. Agarwal, M. F. A'Hearn, M. A. Barucci, J.-L. Bertaux, I. Bertini, G. Cremonese, V. Da Deppo, S. Debei, M. De Cecco, S. Fornasier, M. Fulle, P. J. Gutiérrez, C. Güttler, S. Hviid, W.-H. Ip, J. Knollenberg, J.-R. Kramm, E. Kührt, M. Küppers, L. M. Lara, M. Lazzarin, J. J. Lopez Moreno, F. Marzari, M. Massironi, H. Michalik, G. Naletto, N. Oklay, A. Pommerol, L. Sabau, N. Thomas, C. Tubiana, J.-B. Vincent, and K.-P. Wenzel, "Meter-scale thermal contraction crack polygons on the nucleus of comet 67P/Churyumov-Gerasimenko," Icarus 301, 173-188 (2018).

${ }^{33}$ J.-B. Vincent, D. Bodewits, S. Besse, H. Sierks, C. Barbieri, P. Lamy, R. Rodrigo, D. Koschny, H. Rickman, H. U. Keller, J. Agarwal, M. F. A'Hearn, A.-T. Auger, M. A. Barucci, J.-L. Bertaux, I. Bertini, C. Capanna, G. Cremonese, V. da Deppo, B. Davidsson, S. Debei, M. de Cecco, M. R. El-Maarry, F. Ferri, S. Fornasier, M. Fulle, R. Gaskell, L. Giacomini, O. Groussin, A. Guilbert-Lepoutre, P. GutierrezMarques, P. J. Gutiérrez, C. Güttler, N. Hoekzema, S. Höfner, S. F. Hviid, W.-H. Ip, L. Jorda, J. Knollenberg, G. Kovacs, R. Kramm, E. Kührt, M. Küppers, F. La Forgia, L. M. Lara, M. Lazzarin, V. Lee, C. Leyrat, Z.-Y. Lin, J. J. Lopez Moreno, S. Lowry, S. Magrin, L. Maquet, S. Marchi, F. Marzari, M. Massironi, H. Michalik, R. Moissl, S. Mottola, G. Naletto, N. Oklay, M. Pajola, F. Preusker, F. Scholten, N. Thomas, I. Toth, and C. Tubiana, "Large heterogeneities in comet 67P as revealed by active pits from sinkhole collapse," Nature 523, 63-66 (2015).

${ }^{34}$ M. Pajola, N. Oklay, F. La Forgia, L. Giacomini, M. Massironi, I. Bertini, M. R. El-Maarry, F. Marzari, F. Preusker, F. Scholten, S. Höfner, J.-C. Lee, J.-B. Vincent, O. Groussin, G. Naletto, M. Lazzarin, C. Barbieri, H. Sierks, P. Lamy, R. Rodrigo, D. Koschny, H. Rickman, H. U. Keller, J. Agarwal, M. F. A'Hearn, M. A. Barucci, J.-L. Bertaux, G. Cremonese, V. Da Deppo, B. Davidsson, M. De Cecco, S. Debei, F. Ferri, S. Fornasier, M. Fulle, C. Güttler, P. J. Gutierrez, S. F. Hviid, W.-H. Ip, L. Jorda, J. Knollenberg, J.-R. Kramm, M. Küppers, E. Kürt, L. M. Lara, Z.-Y. Lin, J. J. Lopez Moreno, S. Magrin, H. Michalik, S. Mottola, N. Thomas, and C. Tubiana, "Aswan site on comet 67P/Churyumov-Gerasimenko: Morphology, boulder evolution, and spectrophotometry," Astron. Astrophys. 592, A69 (2016).

${ }^{35}$ N. Attree, O. Groussin, L. Jorda, D. Nébouy, N. Thomas, Y. Brouet, E. Kührt, F. Preusker, F. Scholten, J. Knollenberg, P. Hartogh, H. Sierks, C. Barbieri,
P. Lamy, R. Rodrigo, D. Koschny, H. Rickman, H. U. Keller, M. F. A'Hearn, A.-T. Auger, M. A. Barucci, J.-L. Bertaux, I. Bertini, D. Bodewits, S. Boudreault, G. Cremonese, V. Da Deppo, B. Davidsson, S. Debei, M. De Cecco, J. Deller, M. R. El-Maarry, S. Fornasier, M. Fulle, P. J. Gutiérrez, C. Güttler, S. Hviid, W.-H. Ip, G. Kovacs, J. R. Kramm, M. Küppers, L. M. Lara, M. Lazzarin, J. J. Lopez Moreno, S. Lowry, S. Marchi, F. Marzari, S. Mottola, G. Naletto, N. Oklay, M. Pajola, I. Toth, C. Tubiana, J.-B. Vincent, and X. Shi, "Tensile strength of 67P/Churyumov-Gerasimenko nucleus material from overhangs," Astron. Astrophys. 611, A33 (2018); arXiv:1712.07508 [astro-ph.EP].

${ }^{36}$ S. E. Schröder, S. Mottola, G. Arnold, H.-G. Grothues, R. Jaumann, H. U. Keller, H. Michaelis, J.-P. Bibring, I. Pelivan, A. Koncz, K. Otto, E. Remetean, F. Souvannavong, and B. Dolives, "Close-up images of the final Philae landing site on comet $67 \mathrm{P} /$ Churyumov-Gerasimenko acquired by the ROLIS camera," Icarus 285, 263-274 (2017); arXiv:1701.00685 [astro-ph.EP].

${ }^{37}$ K. A. Otto, K.-D. Matz, S. E. Schröder, R. Parekh, K. Krohn, R. Honda, S. Kameda, R. Jaumann, N. Schmitz, K. Stephan, S. Sugita, E. Tatsumi, T.-M. Ho, A. Koncz, F. Trauthan, Y. Cho, M. Hayakawa, C. Honda, T. Kouyama, M. Matsuoka, T. Morota, S. Mottola, K. Ogawa, F. Preusker, N. Sakatani, H. Sawada, F. Scholten, H. Suzuki, M. Yamada, Y. Yokota, and K. Yoshioka, "Surface roughness of asteroid (162173) Ryugu and comet 67P/Churyumov-Gerasimenko inferred from in situ observations," Mon. Not. R. Astron. Soc. 500, 3178-3193 (2020).

${ }^{38}$ A. La Porta, G. A. Voth, A. M. Crawford, J. Alexander, and E. Bodenschatz, "Fluid particle accelerations in fully developed turbulence," Nature 409, 1017-1019 (2001); arXiv:physics/0011017 [physics.flu-dyn].

${ }^{39} \mathrm{~N}$. T. Ouellette, H. Xu, and E. Bodenschatz, "A quantitative study of threedimensional Lagrangian particle tracking algorithms," Exp. Fluids 40, 301-313 (2006).

${ }^{40} \mathrm{H}$. Xu, "Tracking Lagrangian trajectories in position velocity space," Meas. Sci. Technol. 19, 075105 (2008).

${ }^{41} \mathrm{~B}$. Wieneke, "Volume self-calibration for 3D particle image velocimetry," Exp. Fluids 45, 549-556 (2008).

${ }^{42}$ H. L. Capelo, J. Moláček, M. Lambrechts, J. Lawson, A. Johansen, J. Blum, E. Bodenschatz, and H. Xu, "Observation of aerodynamic instability in the flow of a particle stream in a dilute gas," Astron. Astrophys. 622, A151 (2019); arXiv:1812.01072 [astro-ph.EP].

${ }^{43}$ Y. Skorov, V. Reshetnyk, L. Rezac, Y. Zhao, R. Marschall, J. Blum, and P. Hartogh, "Dynamical properties and acceleration of hierarchical dust in the vicinity of comet 67P/Churyumov-Gerasimenko," Mon. Not. R. Astron. Soc. 477, 4896-4907 (2018).

${ }^{44}$ R. Marschall, Y. Liao, N. Thomas, and J.-S. Wu, "Limitations in the determination of surface emission distributions on comets through modelling of observational data-A case study based on Rosetta observations," Icarus 346, 113742 (2020); arXiv:2004.13199 [astro-ph.EP].

${ }^{45}$ S. Chandrasekhar, Radiative Transfer (Dover, New York, 1960).

${ }^{46}$ C. Bohren and D. Huffman, Absorption and Scattering of Light by Small Particles (John Wiley, Ltd., 1998), pp. 1-530, https://onlinelibrary.wiley. com/doi/pdf/10.1002/9783527618156.fmatter.

${ }^{47}$ P.-E. Wolf and G. Maret, "Weak localization and coherent backscattering of photons in disordered media," Phys. Rev. Lett. 55, 2696-2699 (1985).

${ }^{48}$ E. Akkermans and G. Montambaux, Mesoscopic Physics of Electrons and Photons (Cambridge University Press, 2007), pp. 1-588.

${ }^{49}$ M. J. Gaffey, E. A. Cloutis, M. S. Kelley, and K. L. Reed, "Mineralogy of asteroids," in Asteroids III (University of Arizona Press, 2002), pp. 183-204.

${ }^{50}$ T. Nakamura, T. Noguchi, M. Tanaka, M. E. Zolensky, M. Kimura, A. Tsuchiyama, A. Nakato, T. Ogami, H. Ishida, M. Uesugi, T. Yada, K. Shirai, A. Fujimura, R. Okazaki, S. A. Sandford, Y. Ishibashi, M. Abe, T. Okada, M. Ueno, T. Mukai, M. Yoshikawa, and J. Kawaguchi, "Itokawa dust particles: A direct link between S-type asteroids and ordinary chondrites," Science 333, 1113 (2011).

${ }^{51}$ Y. Shkuratov, V. Kaydash, V. Korokhin, Y. Velikodsky, N. Opanasenko, and G. Videen, "Optical measurements of the Moon as a tool to study its surface," Planet. Space Sci. 59, 1326-1371 (2011).

${ }^{52}$ B. Hapke, Topics in Remote Sensing, 2nd ed. (Cambridge University Press, Cambridge, UK, 1993).

${ }^{53}$ C. Feller, K. Kipfer, H. Capelo, S. Spadaccia, A. Pommerol, and N. Thomas, "Photometric measurements and calibrations in view of the cometary 
physics laboratory experiments," in EPSC-DPS Joint Meeting (Europlanet Science Congress/Division for Planetary Sciences, 2019), Vol. 2019, p. EPSC-DPS2019-1423.

${ }^{54} \mathrm{~A}$. Zoehrer, "Laboratory experiments and numerical modelling of cone penetration tests into various Martian soil analogue materials," Ph.D. dissertation (Technical University Graz, Institute of Soil Mechanics and Foundation Engineering, 2006), a full Ph.D thesis entry.

${ }^{55}$ E. M. R. Fairbairn and F.-J. Ulm, "A tribute to Fernando L. L. B. Carneiro (1913-2001) engineer and scientist who invented the Brazilian test," Mater. Struct. 35, 195-196 (2002).

${ }^{56}$ M. Grott, J. Knollenberg, M. Hamm, K. Ogawa, R. Jaumann, K. A. Otto, M. Delbo, P. Michel, J. Biele, W. Neumann, M. Knapmeyer, E. Kührt, H. Senshu, T. Okada, J. Helbert, A. Maturilli, N. Müller, A. Hagermann, N. Sakatani, S. Tanaka, T. Arai, S. Mottola, S. Tachibana, I. Pelivan, L. Drube, J. B. Vincent, H. Yano, C. Pilorget, K. D. Matz, N. Schmitz, A. Koncz, S. E. Schröder, F. Trauthan, M. Schlotterer, C. Krause, T. M. Ho, and A. Moussi-Soffys, "Low thermal conductivity boulder with high porosity identified on C-type asteroid (162173) Ryugu," Nat. Astron. 3, 971-976 (2019).

${ }^{57}$ M. Grott, J. Knollenberg, B. Borgs, F. Hänschke, E. Kessler, J. Helbert, A. Maturilli, and N. Müller, "The MASCOT radiometer MARA for the Hayabusa 2 mission,” Space Sci. Rev. 208, 413-431 (2017).

${ }^{58}$ W. Sabuga and U. Hammerschmidt, "A new method for the evaluation of thermal conductivity and thermal diffusivity from transient hot strip measurements," Int. J. Thermophys. 16, 557-565 (1995).

${ }^{59}$ P. Tiefenbacher, N. I. Kömle, W. Macher, and G. Kargl, "Influence of probe geometry on measurement results of non-ideal thermal conductivity sensors," Geosci. Instrum., Methods Data Syst. 5, 383-401 (2016).

${ }^{60}$ Z. Zivkovic and F. van der Heijden, "Efficient adaptive density estimation per image pixel for the task of background subtraction," Pattern Recognit. Lett. 27, 773-780 (2006).

${ }^{61}$ D. Schanz, A. Schröder, S. Gesemann, D. Michaelis, and B. Wieneke, "Shake the box: A highly efficient and accurate tomographic particle tracking velocimetry (TOMO-PTV) method using prediction of particle positions," in International Symposium on Particle Image Velocimetry (Illinois Institute of Technology, 2013), Vol. 10, pp. S1-S13.

${ }^{62}$ B. Gundlach, Y. V. Skorov, and J. Blum, "Outgassing of icy bodies in the solar system-I. The sublimation of hexagonal water ice through dust layers," Icarus 213, 710-719 (2011); arXiv:1101.2518 [astro-ph.EP].

${ }^{63}$ A. Pommerol, B. Jost, O. Poch, M. R. El-Maarry, B. Vuitel, and N. Thomas, "The SCITEAS experiment: Optical characterizations of sublimating icy planetary analogues," Planet. Space Sci. 109-110, 106-122 (2015).

${ }^{64}$ M. R. El-Maarry, A. Pommerol, and N. Thomas, "Desiccation of phyllosilicatebearing samples as analog for desiccation cracks on Mars: Experimental setup and initial results," Planet. Space Sci. 111, 134-143 (2015).

${ }^{65}$ A. Pommerol, B. Jost, O. Poch, Z. Yoldi, Y. Brouet, A. Gracia-Berná, R. Cerubini, A. Galli, P. Wurz, B. Gundlach, J. Blum, N. Carrasco, C. Szopa, and N. Thomas, "Experimenting with mixtures of water ice and dust as analogues for icy planetary material. Recipes from the ice laboratory at the University of Bern," Space Sci. Rev. 215, 37 (2019).

${ }^{66}$ O. Poch, A. Pommerol, B. Jost, N. Carrasco, C. Szopa, and N. Thomas, "Sublimation of ice-tholins mixtures: A morphological and spectro-photometric study," Icarus 266, 288-305 (2016).

${ }^{67}$ O. Poch, A. Pommerol, B. Jost, N. Carrasco, C. Szopa, and N. Thomas, "Sublimation of water ice mixed with silicates and tholins: Evolution of surface texture and reflectance spectra, with implications for comets," Icarus 267, 154-173 (2016).

${ }^{68}$ B. Jost, A. Pommerol, O. Poch, Z. Yoldi, S. Fornasier, P. H. Hasselmann, C. Feller, N. Carrasco, C. Szopa, and N. Thomas, "Bidirectional reflectance and VIS-NIR spectroscopy of cometary analogues under simulated space conditions," Planet. Space Sci. 145, 14-27 (2017)

${ }^{69}$ Z. Yoldi, A. Pommerol, B. Jost, O. Poch, J. Gouman, and N. Thomas, "VIS-NIR reflectance of water ice/regolith analogue mixtures and implications for the detectability of ice mixed within planetary regoliths," Geophys. Res. Lett. 42, 6205-6212, https://doi.org/10.1002/2015gl064780 (2015).

${ }^{70}$ M. S. Bessell, "UBVRI passbands," Publ. Astron. Soc. Pac. 102, 1181-1199 (1990).

${ }^{71}$ A. Pommerol, N. Thomas, M. R. El-Maarry, M. Pajola, O. Groussin, A.-T. Auger, N. Oklay, S. Fornasier, C. Feller, B. Davidsson, A. Gracia-Berná, B. Jost, R. Marschall, O. Poch, M. A. Barucci, J.-L. Bertaux, F. La Forgia, H. U. Keller, E. Kührt, S. C. Lowry, S. Mottola, G. Naletto, H. Sierks, C. Barbieri, P. L. Lamy, R. Rodrigo, D. Koschny, H. Rickman, J. Agarwal, M. F. A'Hearn, I. Bertini, S. Boudreault, G. Cremonese, V. Da Deppo, M. De Cecco, S. Debei, C. Güttler, M. Fulle, P. J. Gutierrez, S. F. Hviid, W.-H. Ip, L. Jorda, J. Knollenberg, G. Kovacs, J.-R. Kramm, E. Küppers, L. Lara, M. Lazzarin, J. L. Lopez Moreno, F. Marzari, H. Michalik, F. Preusker, F. Scholten, C. Tubiana, and J.-B. Vincent, "OSIRIS observations of meter-sized exposures of $\mathrm{H}_{2} \mathrm{O}$ ice at the surface of 67P/Churyumov-Gerasimenko and interpretation using laboratory experiments," Astron. Astrophys. 583, A25 (2015).

${ }^{72}$ M. C. De Sanctis, F. Capaccioni, M. Ciarniello, G. Filacchione, M. Formisano, S. Mottola, A. Raponi, F. Tosi, D. Bockelée-Morvan, S. Erard, C. Leyrat, B. Schmitt, E. Ammannito, G. Arnold, M. A. Barucci, M. Combi, M. T. Capria, P. Cerroni, W.-H. Ip, E. Kuehrt, T. B. McCord, E. Palomba, P. Beck, E. Quirico, VIRTIS Team, G. Piccioni, G. Bellucci, M. Fulchignoni, R. Jaumann, K. Stephan, A. Longobardo, V. Mennella, A. Migliorini, J. Benkhoff, J. P. Bibring, A. Blanco, M. Blecka, R. Carlson, U. Carsenty, L. Colangeli, M. Combes, J. Crovisier, P. Drossart, T. Encrenaz, C. Federico, U. Fink, S. Fonti, P. Irwin, Y. Langevin, G. Magni, L. Moroz, V. Orofino, U. Schade, F. Taylor, D. Tiphene, G. P. Tozzi, N. Biver, L. Bonal, J.-P. Combe, D. Despan, E. Flamini, S. Fornasier, A. Frigeri, D. Grassi, M. S. Gudipati, F. Mancarella, K. Markus, F. Merlin, R. Orosei, G. Rinaldi, M. Cartacci, A. Cicchetti, S. Giuppi, Y. Hello, F. Henry, S. Jacquinod, J. M. Rees, R. Noschese, R. Politi, and G. Peter, "The diurnal cycle of water ice on comet 67P/Churyumov-Gerasimenko," Nature 525, 500-503 (2015).

${ }^{73}$ S. Fornasier, S. Mottola, H. U. Keller, M. A. Barucci, B. Davidsson, C. Feller, J. D. P. Deshapriya, H. Sierks, C. Barbieri, P. L. Lamy, R. Rodrigo, D. Koschny, H. Rickman, M. A'Hearn, J. Agarwal, J.-L. Bertaux, I. Bertini, S. Besse, G. Cremonese, V. Da Deppo, S. Debei, M. De Cecco, J. Deller, M. R. El-Maarry, M. Fulle, O. Groussin, P. J. Gutierrez, C. Güttler, M. Hofmann, S. F. Hviid, W.-H. Ip, L. Jorda, J. Knollenberg, G. Kovacs, R. Kramm, E. Kührt, M. Küppers, M. L. Lara, M. Lazzarin, J. J. L. Moreno, F. Marzari, M. Massironi, G. Naletto, N. Oklay, M. Pajola, A. Pommerol, F. Preusker, F. Scholten, X. Shi, N. Thomas, I. Toth, C. Tubiana, and J.-B. Vincent, "Rosetta's comet 67P/Churyumov-Gerasimenko sheds its dusty mantle to reveal its icy nature," Science 354, 1566-1570 (2016).

${ }^{74}$ H. U. Keller and E. Kührt, "Cometary nuclei-From Giotto to Rosetta," Space Sci. Rev. 216, 14 (2020).

${ }^{75}$ C. Feller et al., "Spectro-photometric properties of CoPhyLab's materials," (unpublished). 